\title{
Optimal Bichromatic Plane Spanning Trees For Special Point Sets
}

\author{
by \\ Kimberly Crosbie \\ A Thesis submitted to \\ the Faculty of Graduate Studies and Research \\ in partial fulfilment of \\ the requirements for the degree of \\ Master of Computer Science
}

Carleton University

Ottawa, Ontario, Canada

April 2017

Copyright $(\odot$

2017 - Kimberly Crosbie 


\section{Abstract}

Given a point set $S=R \cup B$, where $R$ is a set of red points and $B$ is a set of blue points, we desire to find $T^{*}$, a minimum weight spanning tree such that every edge has one red endpoint and one blue endpoint and no two edges cross. We call $T^{*}$ a bichromatic plane minimum spanning tree (MinBPST). We say a point set is semi-collinear when the blue points lie on a line and the red points lie on one side of the line. In this thesis, we present an $O\left(|B|^{3}|R|^{2}\right)$ running time algorithm for finding $T^{*}$ on a set of semi-collinear points. We also discuss an implementation of this algorithm. Additionally, we describe changes that can be made to the algorithm presented to solve other related problems. Finally, we describe properties of $T^{*}$ on semi-collinear point sets. 


\section{Acknowledgments}

I could not have completed this thesis without the support of many people. To my supervisors Michiel Smid and Anil Maheshwari, thank you for your guidance, for your instruction and for sharing your love of algorithms and computational geometry with me. To Ahmad Biniaz, thank you for introducing me to this problem and for your guidance throughout my research. To my defence committee members Pat Morin and Jean-Lou De Carufel, thank you for your helpful comments and suggestions. My thesis is better because of them. To the members of the computational geometry lab, thank you for your camaraderie and for all of the chocolate. Working in the lab was always a joy. To my parents, thank you for being a constant source of encouragement, comfort and support. You're the best parents a girl could ask for and definitely my favourites. To the rest of my family, thank you for your friendship and unwavering love. To my friends, thank you for being there when I needed you the most. In particular, to Anna and Genevieve, thank you for your listening ears, your prayers and your friendship. To Teri, thank you for understanding this thing called graduate school with me and for your encouragement throughout my degree. Above all, thank you God for being my sustainer and the constant in my life. I truly am blessed beyond belief. 


\section{Table of Contents}

Abstract $\quad$ ii

Acknowledgments $\quad$ iii

Table of Contents $\quad$ iv

List of Tables $\quad$ vi

List of Figures $\quad$ vii

1 Introduction 1

1.1 Problem Statement . . . . . . . . . . . . . . . . . . 2

1.2 Related Work . . . . . . . . . . . . . . . . . . . 3

1.2.1 Minimum Spanning Trees on Non-Coloured Point Sets . . . . . . 4

1.2.2 Minimum Bichromatic Spanning Trees . . . . . . . . . . . . . . . 5

1.3 Contributions and Thesis Organization . . . . . . . . . . . 7

2 MinBPST for Semi-Collinear Points $\quad 8$

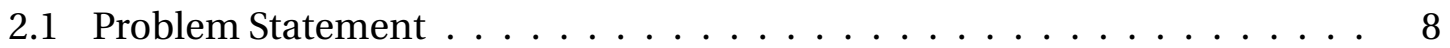

2.2 A Dynamic Programming Algorithm . . . . . . . . . . . . . . 9

2.2.1 Subproblem $A(i, j, u, v$, ex, ex, con $) \ldots \ldots \ldots \ldots$

2.2.2 Subproblem $A(i, j, u, v, i n$, ex, con $) \ldots \ldots \ldots$

2.2.3 Subproblem $A(i, j, u, u, i n, i n, n c o) \ldots \ldots \ldots \ldots$ 
2.2.4 Subproblem $A(i, j, u, v$, ex, ex,$n c o) \ldots \ldots \ldots \ldots$

2.2.5 Subproblem $A(i, j, u, v, i n, e x, n c o) \ldots \ldots \ldots \ldots$

2.2.6 The Top-Level Problem _ . . . . . . . . . . . . . . . . . . . 19

2.2 .7 Base Cases . . . . . . . . . . . . . . . . . . . . . . . 19

2.2.8 Running Time Analysis . . . . . . . . . . . . . . . . . . 23

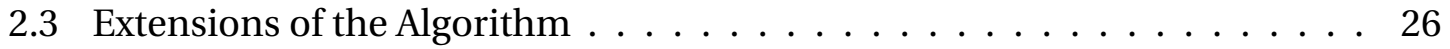

2.4 Implementation of the Algorithm $\ldots \ldots \ldots \ldots \ldots$

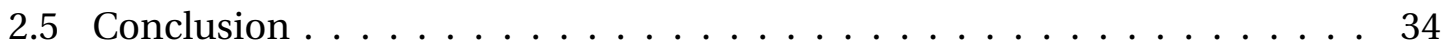

3 Properties of MinBPST on Semi-Collinear Points 35

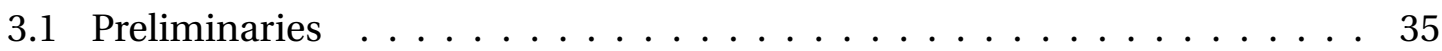

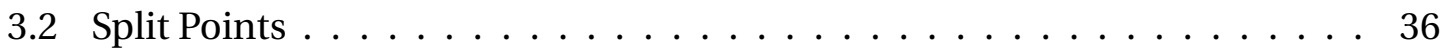

3.3 Obtuse Triangles . . . . . . . . . . . . . . . . 38

3.4 Nonexistence of $\ell^{\prime}$-Crossing Edges . . . . . . . . . . . . . . . 40

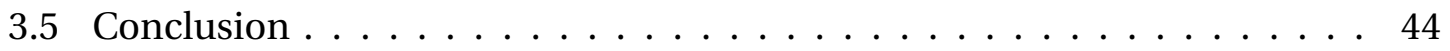

4 Conclusion and Future Work $\quad 45$

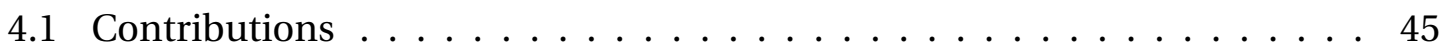

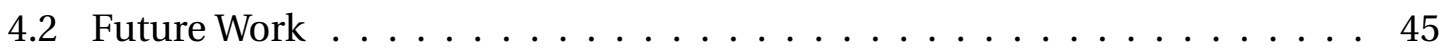

$\begin{array}{ll}\text { List of References } & 48\end{array}$ 


\section{List of Tables}

1 Summary of results for finding MinBPSTs on special point sets $\ldots \ldots \ldots$ 


\section{List of Figures}

1 (a) minimum bichromatic plane spanning tree (b) maximum bichromatic plane spanning tree (c) minimum bottleneck bichromatic plan spanning tree (d) maximum bottleneck bichromatic plane spanning tree . . . . . . . 3

2 An example of a minimum bichromatic spanning tree that is not plane. . . 5

3 An example where the modified version of Kruskal's algorithm does not

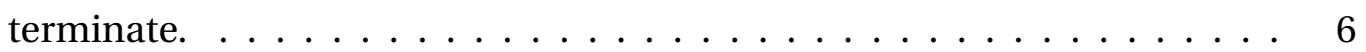

4 An illustration to show that the greedy planar algorithm does not necessarily find the MinBPST. (a) Greedy Algorithm Solution. (b) Optimal Solu-

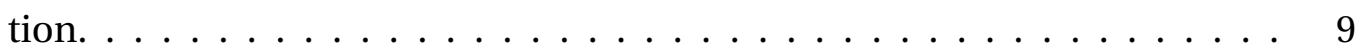

$5 \quad$ Illustration for solving $A(i, j, u, v$, ex, ex,$n c o) \ldots \ldots \ldots \ldots \ldots$

$6 \quad$ Illustration for solving $A(i, j, u, v$, ex, ex, con $) \ldots \ldots \ldots \ldots$. . . . . 12

$7 \quad$ Illustration for solving $A(i, j, u, v, i n$, ex, con $) \ldots \ldots \ldots \ldots \ldots$

8 Illustration for solving $A(i, j, u, u$, in, in, nco $\ldots \ldots \ldots \ldots \ldots$

$9 \quad$ Illustration for solving $A(i, j, u, v, \mathrm{ex}, \mathrm{ex}, \mathrm{nco}) \ldots \ldots \ldots \ldots$

10 Illustration for solving $A(i, j, u, v$, in, ex, nco $\ldots \ldots \ldots \ldots \ldots$

11 Illustration of the recurrence tree for $A(\cdot) \ldots \ldots \ldots \ldots$

12 Illustration of Base Case $1 \ldots \ldots \ldots \ldots$

13 Illustration of Base Case $2 \ldots \ldots \ldots \ldots \ldots \ldots$

14 Illustration of Base Case $3 \ldots \ldots \ldots \ldots \ldots \ldots \ldots$

15 Illustration of Base Case $4 \ldots \ldots \ldots \ldots \ldots \ldots$ 
16 Illustration of Base Case $5 \ldots \ldots \ldots \ldots$

17 An example of the implementation with a generated minimum bichromatic plane spanning tree . . . . . . . . . . . . . . . 30

18 An example of the implementation with a generated maximum bichromatic plane spanning tree . . . . . . . . . . . . . . . 31

19 An example of the implementation with a generated minimum bottleneck bichromatic plane spanning tree . . . . . . . . . . . . . . . . . 32

20 An example of the implementation with a generated maximum bottleneck bichromatic plane spanning tree . . . . . . . . . . . 33

21 Illustration of the definition of rightmost and leftmost edges. Here, $r_{1} b$ is the leftmost edge with endpoint $b$ whereas $r_{5} b$ is the rightmost edge with

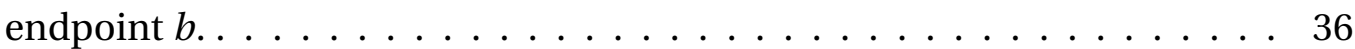

22 Illustration of split points. . . . . . . . . . . . . . . 37

23 Illustration of proof of Lemma 3.3.1. Here we would add the edge $r^{\prime} b_{k+1}$ and remove the edge $r b_{j} \ldots \ldots \ldots \ldots \ldots \ldots$

24 Illustration of proof of Lemma 3.4.1. (a) Case 1: $r^{*}$ is $\bar{r}$-connected to $b_{i}$, but not $b_{j}$. (b) Case 2: $r^{*}$ is $\bar{r}$-connected to $b_{j}$, but not $b_{i}$. . . . . . .

25 Illustration of proof of Lemma 3.4.1 case 2. (a) Case a: $r^{\prime}$ and $r^{\prime \prime}$ lie on the same side of $\ell^{\prime}$. (b) Case b: $r^{\prime \prime}$ lies on the other side of $\ell^{\prime}$ than $b^{\prime \prime}$ and $r^{\prime \prime}$ is higher than $r^{\prime}$. (c) Case c: $r^{\prime \prime}$ lies on the other side of $\ell^{\prime}$ than $b^{\prime \prime}$ and $r^{\prime}$ is higher than $r^{\prime \prime} \ldots \ldots \ldots \ldots \ldots \ldots \ldots \ldots \ldots$

26 An illustration to show a potential approximation ratio between the MinBPST and the minimum bichromatic spanning tree. (a) MinBPST (b) Minimum Bichromatic Spanning Tree . . . . . . . . . . . . . . . . . . . . 47 


\section{Chapter 1}

\section{Introduction}

Computational geometry is a branch of computer science that studies algorithms concerning geometric problems. Within computational geometry, one can design algorithms that take a set of points in the Euclidean plane as input. Sometimes these points may each have a colour associated with them. We call a set of points that has points of only two different colours a bichromatic point set.

A Euclidean graph is a set of points and a set of straight line segments that connect these points together. In a Euclidean graph, each straight line segment must connect a single point to another single point. The points are commonly referred to as vertices and the line segments are commonly referred to as edges. These edges all have a value associated with them, which is the Euclidean length of the edge. We call this value the weight of the edge.

A complete graph is a graph in which each vertex is connected by a single edge to every other vertex. A spanning tree is a Euclidean graph that connects all of the input vertices together without creating any cycles. A cycle occurs when there is a path in the graph that connects a vertex to itself. A minimum spanning tree is a spanning tree that minimizes the total length of the edges in the spanning tree. Finding a minimum spanning tree from a given set of points is an example of an algorithm that takes a set of points in the Euclidean plane as input. One can use a greedy approach to find a 
minimum spanning tree.

A bichromatic spanning tree is a spanning tree on a bichromatic point set where every edge connects two vertices of different colours. A minimum bichromatic spanning tree is a bichromatic spanning tree that minimized the total weight of the edges. A greedy approach can also be used to find the minimum bichromatic spanning tree.

A graph is called plane if no two edges in the graph cross. A minimum bichromatic plane spanning tree is a bichromatic plane spanning tree that minimizes the total weight of the edges. A greedy approach can not be used to find a minimum bichromatic spanning tree. Thus, a interesting problem is finding the minimum bichromatic plane spanning tree efficiently. In this thesis we study bichromatic plane spanning trees on a special set of bichromatic points.

We now outline the remainder of this chapter. Section 1.1 formally defines the problems we study in this thesis. Section 1.2 discusses related work. Section 1.3 describes our contributions and the organization of the remainder of the thesis.

\subsection{Problem Statement}

A bichromatic plane spanning tree (BPST) on a set of bichromatic (red and blue) points is a spanning tree such that all edges in the tree have one red endpoint and one blue endpoint and no two edges cross. We define the weight of a BPST to be the total length of all of the edges in the tree. Of all possible BPSTs on a given point set, a minimum bichromatic plane spanning tree (MinBPST) is a BPST with minimum weight.

Of all possible BPSTs on a given bichromatic point set, a maximum bichromatic plane spanning tree, (MaxBPST), is a BPST with maximum weight.

A minimum bottleneck bichromatic plane spanning tree on a set of bichromatic points is a spanning tree that minimizes the length of the longest edge in the tree.

A maximum bottleneck bichromatic plane spanning tree on a set of bichromatic 


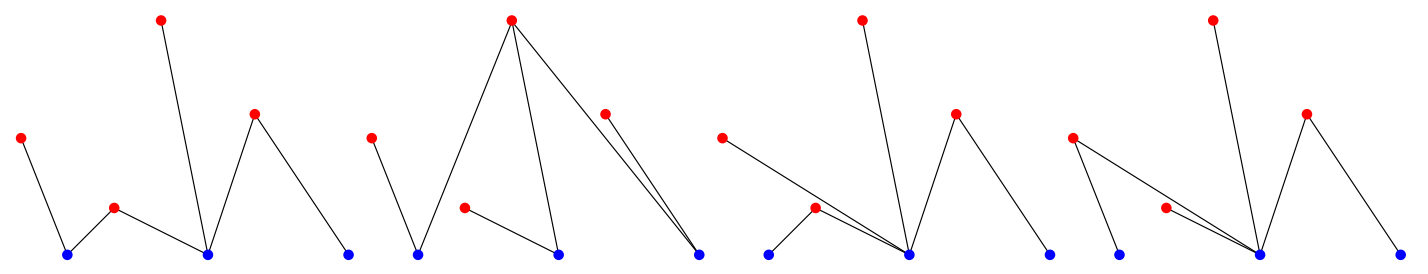

(a) (b) (c)

Figure 1: (a) minimum bichromatic plane spanning tree (b) maximum bichromatic plane spanning tree (c) minimum bottleneck bichromatic plan spanning tree (d) maximum bottleneck bichromatic plane spanning tree

points is a spanning tree that maximizes the length of the smallest edge in the tree.

Figure 1 gives an example of each of the different kinds of bichromatic plane spanning trees.

In this thesis, we say a set of bichromatic points is semi-collinear if all of the blue points lie on a line and all of the red points lie on one side of the line. Without loss of generality, we assume the line that the blue points lie on is horizontal and the red points lie above this line. We assume no three points, where two of the points are red and one of the points is blue, are collinear.

Our main problem is as follows: given a set of bichromatic semi-collinear points, find $T^{*}$, a MinBPST. Similarly we desire to find a MaxBPST, a minimum bottleneck bichromatic plane spanning tree, and a maximum bottleneck bichromatic plane spanning tree on a given set of bichromatic semi-collinear points.

\subsection{Related Work}

Many problems concerning bichromatic (red-blue) point sets have been studied in the field of computational geometry. Kaneko and Kano [1] provide an overview of some of these results. However, several results for problems on bichromatic point sets have been improved upon and discovered since the publication of [1]. 


\subsubsection{Minimum Spanning Trees on Non-Coloured Point Sets}

A classical problem in computational geometry is to find a Euclidean minimum spanning tree, that is the spanning tree with minimum total edge weight, on a point set. For the purpose of this thesis, we say the weight of a spanning tree is the total Euclidean length of all the edges in the spanning tree.

Several algorithms are known for finding the minimum spanning tree (MST) on a point set that is not bichromatic ( [2-4]). Of particular interest is Kruskal's algorithm [4] and Boruvka's algorithm ( $[3,5])$.

Kruskal's algorithm [4] uses a greedy approach to find the MST. Specifically, the algorithm sorts all of the potential edges by weight and adds them one by one, in order of increasing weight, only adding an edge if it does not create a cycle in the graph. The algorithm continues in this way until all of the points are added to the spanning tree.

Boruvka's algorithm $[3,5]$ starts by connecting each point to the nearest other point, creating a forest. We refer to each tree in the forest as a component. The nearest component, $C_{a}$, to component $C_{b}$ is the component that has a vertex with the minimum Euclidean distance from one of the vertices $C_{b}$. The algorithm then connects these components in a similar manner, connecting a component to its nearest other component. This process continues until a spanning tree is formed.

A Delaunay triangulation on a set of points, $P$, in general position, is a triangulation such that no points in $P$ lie in the circumcircle of any of the triangles in the triangulation. The edges in the Euclidean minimum spanning tree are edges in the Delaunay triangluation [6]. Hence, on a non-coloured set of points a Euclidean minimum spanning tree does not contain any crossings and is therefore plane [7]. 


\subsubsection{Minimum Bichromatic Spanning Trees}

A minimum bichromatic spanning tree may contain crossings. Figure 2 shows an example of such a tree. Recently, Biniaz et al. [8] showed that the minimum bichromatic spanning tree can be found in $\Theta(n \log n)$ time. Their approach uses a modified version of Boruvka's algorithm. The resulting minimum bichromatic spanning tree may or may not be plane.

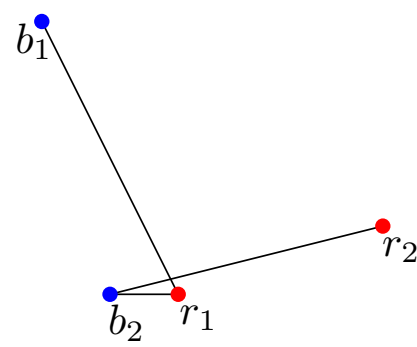

Figure 2: An example of a minimum bichromatic spanning tree that is not plane.

We now turn our attention to finding the minimum bichromatic plane spanning tree, $T^{*}$. In order to find $T^{*}$, one could consider using a greedy approach. Grantson et al. [9] considered a modified version of Kruskal's algorithm. In particular, edges were only added if the resulting graph did not contain cycles or edge crossings. It should be noted that they also demonstrated that the resulting tree is not necessarily the optimal one. Borgelt et al. [10] also gave an example of a particular bichromatic point set where this modified version of Kruskal's algorithm is unable to produce a spanning tree. Their example shows that it is possible to reach a stage in the algorithm when a point of one colour is unable to connect to any point of the other colour without creating a crossing. Figure 3 replicates their example where the point $b_{9}$ is unable to connect to any blue points without creating a crossing.

Borgelt et al. [10] showed that finding $T^{*}$ in the general case is an NP-Hard problem. They proved that this problem is NP-Hard by a reduction from the planar 3-SAT 


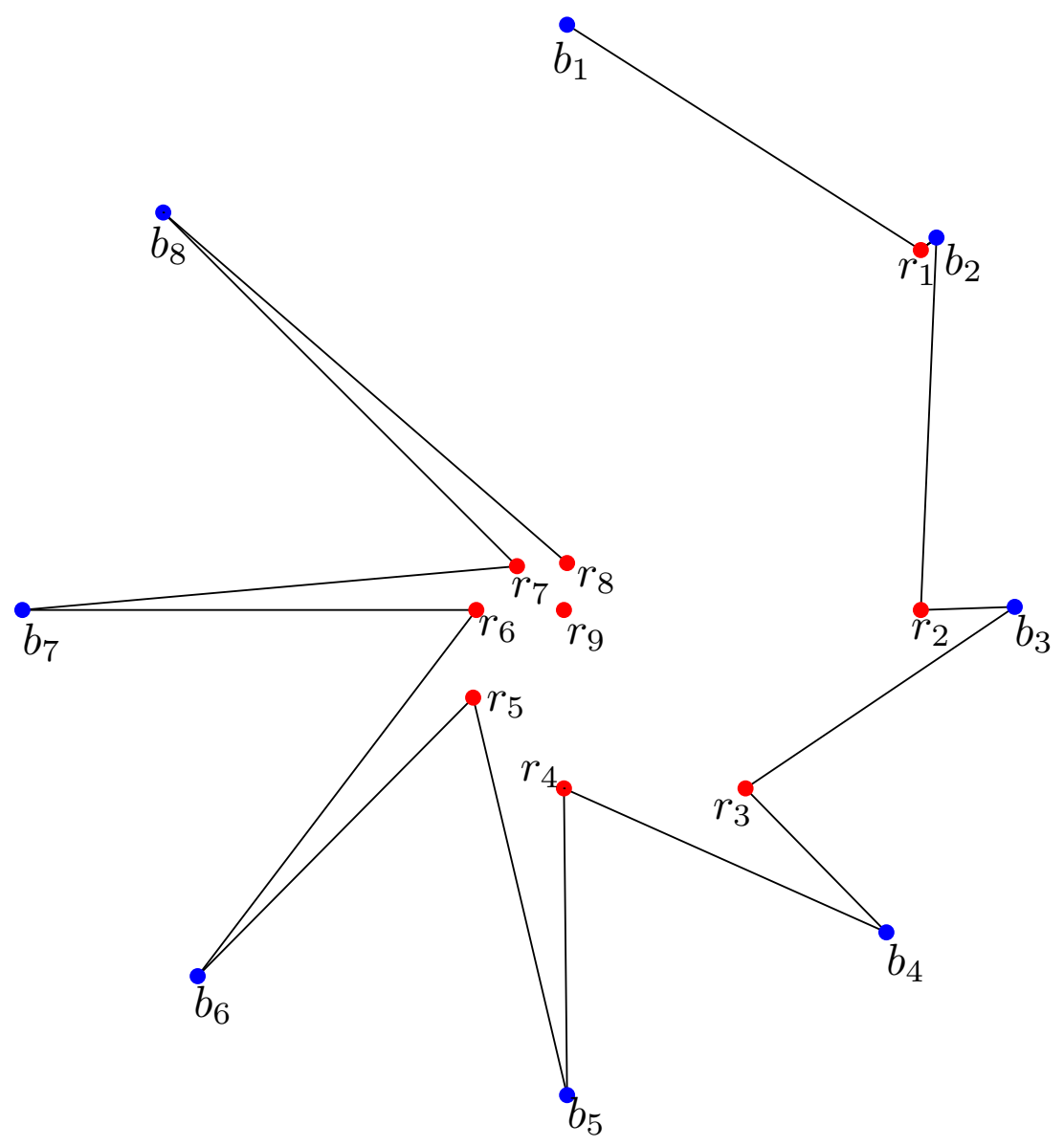

Figure 3: An example where the modified version of Kruskal's algorithm does not terminate.

problem [11].

Since finding the MinBPST $T^{*}$ is an NP-Hard problem, approximation algorithms that find a planar bichromatic spanning tree within a certain factor of $T^{*}$ are of interest. Currently, the best known result is an $\mathrm{O}(\sqrt{n})$-approximation algorithm presented by Borgelt et al. [10]. The running time of the algorithm is $O(n \log n \log \log n)$.

In their paper, Borgelt et al. [10] discuss several algorithms for finding the MinBPST, $T^{*}$, on special point sets. Table 1 summarizes their results and includes our result (last 


\begin{tabular}{|c|c|c|c|}
\hline Size of $B$ and $R$ & Positioning of Points & Time Complexity & Reference \\
$|B|=n,|R|=m, m \leq n$ & convex position & $O\left(n m^{2}\right)$ & {$[10]$} \\
$|B|=n-2,|R|=2$ & general & $O(n \log n)$ & {$[10]$} \\
$|B|=n-k,|R|=k$ & general & $n^{O\left(k^{5}\right)}$ & {$[10]$} \\
$|B|=n,|R|=m$ & semi-collinear & $O\left(n^{3} m^{2}\right)$ & Theorem 2.2.1 \\
\hline
\end{tabular}

Table 1: Summary of results for finding MinBPSTs on special point sets

row; semi-collinear points).

\subsection{Contributions and Thesis Organization}

In this thesis, we study the special case of finding the MinBPST, $T^{*}$, on bichromatic semi-collinear point sets. Chapter 2 describes an $\mathrm{O}\left(|B|^{3}|R|^{2}\right)$ algorithm, and its implementation, for finding the MinBPST in this case. It then describes how the algorithm can be adapted to find a MaxBPST, a minimum bottleneck bichromatic plane spanning tree, and a maximum bottleneck bichromatic plane spanning tree on a set of bichromatic semi-collinear points. Chapter 3 looks at the properties that $T^{*}$ has when it is on a set of semi-collinear points. Chapter 4 summarizes the thesis and discusses possible future work. 


\section{Chapter 2}

\section{MinBPST for Semi-Collinear Points}

In this chapter we consider a set $B$ of blue points that lie on a line and a set $R$ of red points that lie on one side of the line. This chapter describes how to go about finding the MinBPST for such a point set. We show, by an example, that a greedy algorithm will not necessarily find the MinBPST. We then describe a dynamic programming algorithm that finds the MinBPST in $\mathrm{O}\left(|B|^{3}|R|^{2}\right)$ time. Next we show how this algorithm can be adapted to find a maximum bichromatic plane spanning tree, a minimum bottleneck bichromatic plane spanning tree and a maximum bottleneck bichromatic plane spanning tree. Finally, we describe an implementation of that algorithm.

\subsection{Problem Statement}

We consider the case where the blue points are on a line, $\ell$, and the red points are on one side of $\ell$. Without loss of generality, we assume that the line $\ell$ is horizontal. Formally, we have a set $B$ of $n$ consecutive blue points $b_{1}, \ldots, b_{n}$ that lie on a line $\ell$ and a set $R$ of $m$ red points that lie on one side of the line. Given such a point set we desire to find $T^{*}$, the minimum bichromatic plane spanning tree of the point set $B \cup R$.

First, we show by example that a greedy planar algorithm does not necessarily produce the optimal solution. Figure 4 gives a specific example. Figure 4 (a) shows the PBST 


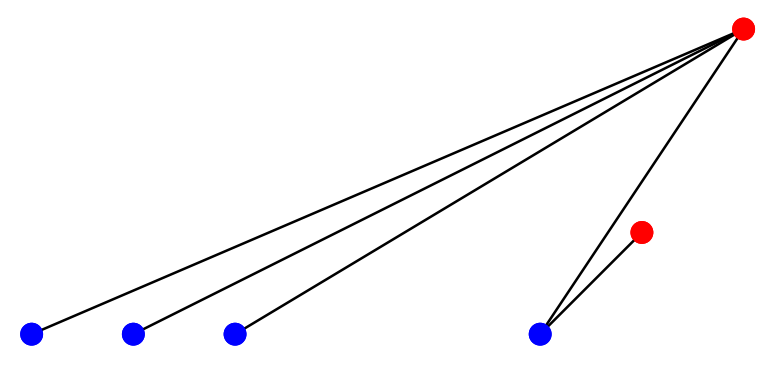

(a)

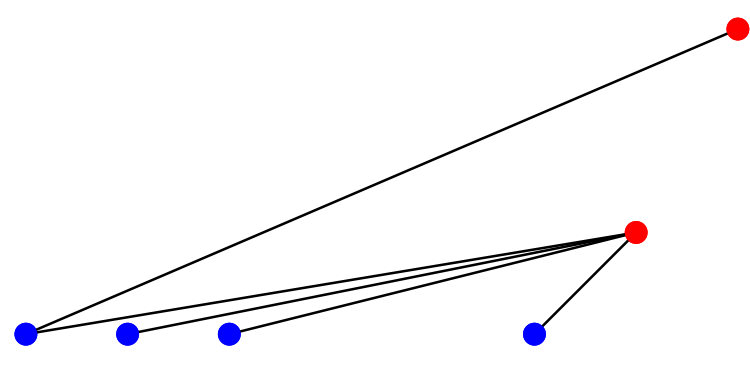

(b)

Figure 4: An illustration to show that the greedy planar algorithm does not necessarily find the MinBPST. (a) Greedy Algorithm Solution. (b) Optimal Solution.

that is produced by running a greedy planar algorithm whereas the right figure shows the actual MinBPST. Thus, in general, a greedy approach cannot be used to find $T^{*}$ on semi-collinear points.

\subsection{A Dynamic Programming Algorithm}

In this section we describe a top-down dynamic programming algorithm for finding the MinBPST that runs in $\mathrm{O}\left(|B|^{3}|R|^{2}\right)$ time. Since we are using a top-down dynamic programming algorithm, we maintain a table of our results. We initialize this table so that every entry is -1 . When we encounter a subproblem we check the corresponding table entry. If the table entry contains a positive value, then we have already solved that subproblem and we need only return that value. Otherwise, we solve the subproblem recursively.

We define the problem $A\left(i, j, u, v, u^{\prime}, v^{\prime}, w\right)$ to be the problem of computing the weight of the MinBPST for a set of blue points, $B_{A}$, and a set of red points, $R_{A}$ where:

- $i$ and $j$, with $i \leq j$ are indices of two blue points such that $B_{A}=\left\{b_{i}, \ldots, b_{j}\right\}$.

- $u$ and $v$ are the indices of two red points such that $R_{A}$ contains the points that lie to the right side of the line segment $r_{u} b_{i}$, to the left side of the line segment $r_{\nu} b_{j}$ 
and have a smaller $y$-coodinate than both $r_{u}$ and $r_{\nu}$.

- $u^{\prime}$ takes a value in the set $\{i n, e x\}$. If $u^{\prime}=i n$ then $r_{u}$ is included in $R_{A}$. If $u^{\prime}=e x$ then $r_{u}$ is excluded from $R_{A}$.

- $v^{\prime}$ takes a value in the set $\{i n, e x\}$. If $v^{\prime}=i n$ then $r_{v}$ is included in $R_{A}$. If $v^{\prime}=e x$ then $r_{\nu}$ is excluded from $R_{A}$.

- $w$ takes a value in the set $\{c o n, n c o\}$. Where $c o n$ stands for connected and $n c o$ stands for not connected.

The output of the problem $A\left(i, j, u, v, u^{\prime}, v^{\prime}, w\right)$ satisfies the following:

- Rule 1: If $u^{\prime}=$ in (resp. $v^{\prime}=$ in), then we have the constraint on $A(\cdot)$ that $r_{u} b_{i}$ (resp. $r_{v} b_{j}$ ) should be part of the solution, but $A(\cdot)$ returns the total weight of new edges, i.e. the weight of a MinBPST minus $\left|r_{u} b_{i}\right|$ (resp. $\left.\left|r_{v} b_{j}\right|\right)$.

- Rule 2: If $u^{\prime}=v^{\prime}=i n$, then $u=v$, and consequently $r_{u}=r_{\nu}$. Thus, every time we call $A(\cdot)$ with $u^{\prime}=v^{\prime}=i n$, we ensure that $u=v$.

- If $w=c o n$, then the output is the weight of the MinBPST for $B_{A} \cup R_{A}$. Note here that the MinBPST must follow Rule 1.

- If $w=n c o$ and $u^{\prime}=v^{\prime}=i n$, then the output is the weight of the MinBPST for $B_{A} \cup R_{A}$ that contains $\left\{b_{i} r_{u}, b_{j} r_{v}\right\}$.

- If $w=n c o$ and $u^{\prime}=v^{\prime}=e x$, then the output is the weight of two vertex-disjoint bichromatic trees $T_{i}$ and $T_{j}$ that $\operatorname{span} B_{A} \cup R_{A}$, where $T_{i} \cup T_{j}$ is plane, $b_{i}$ is a vertex of $T_{i}, b_{j}$ is a vertex of $T_{j}$ and the total weight of $T_{i} \cup T_{j}$ is minimized.

- If $w=n c o, u^{\prime}=e x$ and $v^{\prime}=i n$, then we find two vertex-disjoint bichromatic trees $T_{i}$ and $T_{j}$ that span $B_{A} \cup R_{A}$, where $T_{i} \cup T_{j}$ is plane, $b_{i}$ is a vertex of $T_{i}, b_{j}$ is a vertex 
of $T_{j}, T_{j}$ contains the edge $b_{j} r_{\nu}$ and the total weight of $T_{i} \cup T_{j}$ is minimized. The output is then the weight of $T_{i} \cup T_{j}-\left|b_{j} r_{v}\right|$.

- If $w=n c o, u^{\prime}=i n$ and $v^{\prime}=e x$, then we find two vertex-disjoint bichromatic trees $T_{i}$ and $T_{j}$ that span $B_{A} \cup R_{A}$, where $T_{i} \cup T_{j}$ is plane, $b_{i}$ is a vertex of $T_{i}, b_{j}$ is a vertex of $T_{j}, T_{i}$ contains the edge $b_{i} r_{u}$ and the total weight of $T_{i} \cup T_{j}$ is minimized. The output is then the weight of $T_{i} \cup T_{j}-\left|b_{i} r_{u}\right|$.

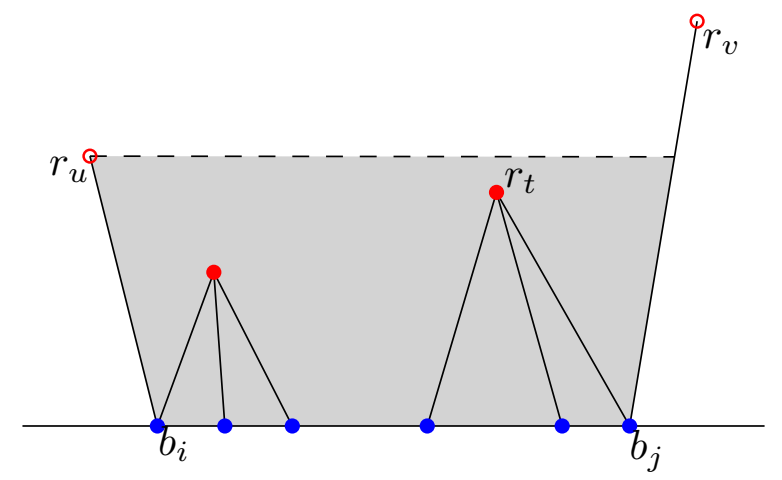

Figure 5: Illustration for solving $A(i, j, u, v$, ex $, e x, n c o)$

Figure 5 gives an example of finding the MinBPST when $u^{\prime}=e x, v^{\prime}=e x$ and $w=$ nco. We have eight possible configurations for $A(\cdot)$ depending on the values of $u^{\prime}, v^{\prime}$, and $w$. In every step of solving $A(\cdot)$ the size of the problem is reduced. Therefore, the algorithm terminates.

\subsubsection{Subproblem $A(i, j, u, v, e x, e x, c o n)$}

Refer to Figure 6. Since $u^{\prime}=e x, r_{u}$ is not contained in $R_{A}$. Similarly, since $v^{\prime}=e x, r_{\nu}$ is not contained in $R_{A}$. Since $w=c o n$, our solution should connect $b_{i}$ and $b_{j}$ by a path. Take the topmost red point in $R_{A}$ and call it $r_{t}$. In an optimal solution, $r_{t}$ is connected by an edge to one or more points in $\left\{b_{i}, \ldots, b_{j}\right\}$. Let $b_{k}$, where $i \leq k \leq j$, be the leftmost blue point adjacent to $r_{t}$. Since no blue points to the left of $b_{k}$ can be connected to any 
red points to the right of the edge $r_{t} b_{k}$ without creating an edge crossing and vice versa, we have split the problem into two independent subproblems.

In the left subproblem, since $b_{k}$ is the leftmost blue point adjacent to $r_{t}$, we exclude $r_{t}$ from the subproblem. The solution to this subproblem must be a single spanning tree that includes both $b_{k}$ and $b_{i}$, thus we still have $w=$ con. In the right subproblem, $r_{t}$ could be adjacent to another blue point, hence it should be included in the right subproblem. We still have $w=$ con in this subproblem since our solution should connect $b_{k}$ to $b_{j}$ by a path. $r_{u}$ and $r_{v}$ remain excluded from $R_{A}$. Therefore, we obtain the following:

$$
\begin{aligned}
A(i, j, u, v, \text { ex }, \text { ex }, \text { con }) & =\min \left\{\left|r_{t} b_{k}\right|+A(i, k, u, t, \text { ex }, \text { ex }, \text { con })\right. \\
& +A(k, j, t, v, i n, \text { ex }, \text { con }): i \leq k \leq j\}
\end{aligned}
$$

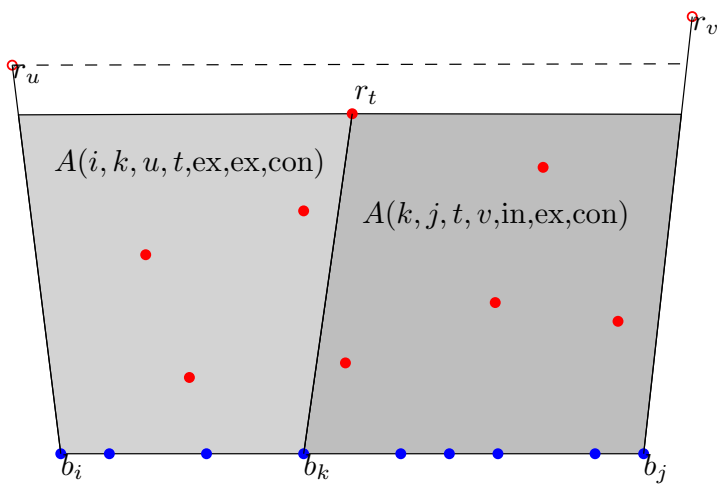

Figure 6: Illustration for solving $A(i, j, u, v$, ex, ex, con $)$

By rule 1 , the solution to $A(i, j, t, v, i n, e x, c o n)$ only returns the weight of the new edges added, hence we do not double count $\left|r_{t} b_{k}\right|$. We try all $j-i+1$ choices for $k$ $(i \leq k \leq j)$ and find the $k$ that minimizes $A(i, j, u, v, e x, e x, c o n)$. 


\subsubsection{Subproblem $A(i, j, u, v, i n, e x, c o n)$}

Refer to Figure 7. In $A(i, j, u, v, i n, e x, c o n), r_{u}$ is included in $R_{A}$ and $r_{v}$ in excluded from $R_{A}$. Hence, the solution should include the edge $r_{u} b_{i}$, but by rule 1 , the value returned by $A(\cdot)$ should not include $\left|r_{u} b_{i}\right|$. We now consider two cases: the case where $r_{u}$ is only adjacent to one blue point, that is $b_{i}$, and the case where $r_{u}$ is adjacent to at least one blue point other than $b_{i}$.

In the case when $r_{u}$ is only adjacent to one blue point, $b_{i}$, we obtain the following solution:

$$
A(i, j, u, v, i n, e x, c o n)=A(i, j, u, v, e x, e x, c o n)
$$

In the case when $r_{u}$ is adjacent to at least one blue point other than $b_{i}$, let $b_{k}$ be the rightmost blue point that is adjacent to $r_{u}$ in an optimal solution, where $i+1 \leq k \leq j$. See Figure 7 for an example. Since $r_{u}$ is the topmost point in $R_{A}$, the edge $r_{u} b_{k}$ splits the problem into two independent subproblems, one to the left of the edge and one to the right of the edge. In the left subproblem, $r_{u}$ should still be included since it is possible for it to be adjacent to more blue points. Since $b_{i}$ and $b_{k}$ are now connected by a path the solution to the left subproblem should not connect them, otherwise a cycle would form. In the right subproblem, since $b_{k}$ is the rightmost blue point that is adjacent to $r_{u}, r_{u}$ should be excluded because $r_{u}$ cannot be connected to any other blue points. $b_{k}$ and $b_{j}$ still need to be connected by a path, thus the solution should connect them. $r_{\nu}$ remains excluded from $R_{A}$. Therefore we have:

$$
\begin{aligned}
A(i, j, u, v, i n, e x, c o n) & =\min \left\{\left|r_{u} b_{k}\right|+A(i, k, u, u, i n, i n, n c o)\right. \\
& +A(k, j, u, v, \text { ex }, \text { ex }, \text { con }): i+1 \leq k \leq j\}
\end{aligned}
$$

We note that here Rule 2 is satisfied. We try all $j-i$ possible values for $k(i+1 \leq k \leq j)$ and choose the one that minimizes $A(i, j, u, v, i n, e x, c o n)$. Between the two cases we pick the one that minimizes $A(\cdot)$. 


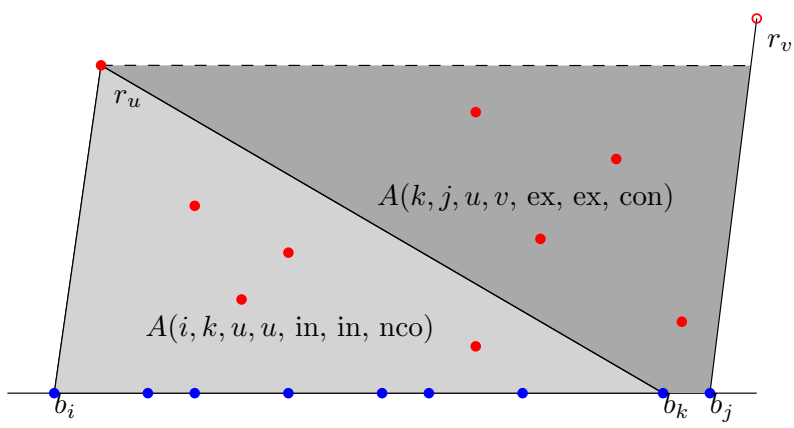

Figure 7: Illustration for solving $A(i, j, u, v, i n, e x$, con $)$

\subsubsection{Subproblem $A(i, j, u, u, i n, i n, n c o)$}

Refer to Figure 8. We consider two cases. In the first case, $r_{u}$ is adjacent to only the two blue points, $b_{i}$ and $b_{j}$. In the second case, $r_{u}$ is connected by an edge to at least one blue point that is not $b_{i}$ or $b_{j}$.

In the case where $r_{u}$ is adjacent to only the two blue points, $b_{i}$ and $b_{j}, r_{u}$ should be excluded from the new problem since it cannot be connected to any other blue points. Then we have:

$$
A(i, j, u, u, i n, i n, n c o)=A(i, j, u, u, e x, e x, n c o)
$$

In the case where $r_{u}$ is connected by an edge to at least one blue point that is not $b_{i}$ or $b_{j}$, let $b_{k}$ be the leftmost blue point connected to $r_{u}$ in an optimal solution with $i+1 \leq k \leq j-1$. The edge $r_{u} b_{k}$ splits the problem into two independent subproblems. In the left subproblem, $r_{u}$ should not be included in $R_{A}$ since it cannot be adjacent to any other blue points because $b_{k}$ is the leftmost blue point adjacent to $r_{u}$. In the right subproblem, $r_{u}$ could be adjacent to another blue point, thus it should be included in the subproblem. See Figure 8 for an example illustration. In both subproblems, it is still 


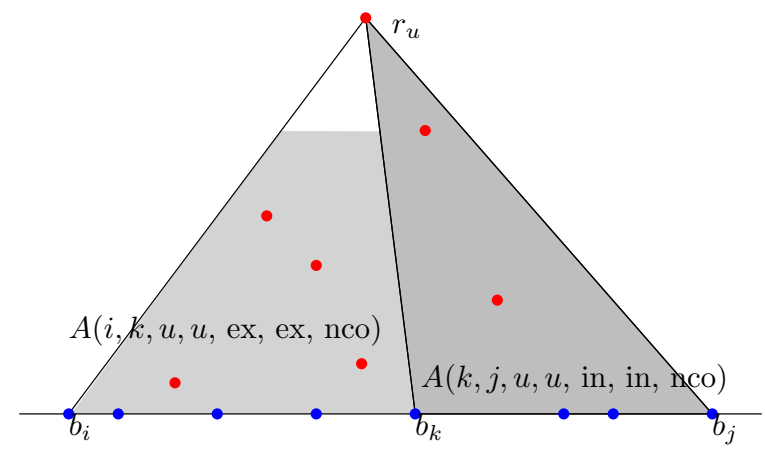

Figure 8: Illustration for solving $A(i, j, u, u$, in, in, nco)

the case that $w=n c o$. Thus we obtain the following:

$$
\begin{aligned}
A(i, j, u, u, i n, i n, n c o) & =\min \left\{\left|r_{u} b_{k}\right|+A(i, k, u, u, \text { ex }, \text { ex }, n c o)\right. \\
& +A(k, j, u, u, i n, i n, n c o): i+1 \leq k \leq j-1\}
\end{aligned}
$$

There are $j-i-1$ possible values for $k(i+1 \leq k \leq j-1)$. We iterate over all of them and pick the one that minimizes $A(i, j, u, u, i n, i n, n c o)$. Between the two cases, we pick the one that minimizes $A(i, j, u, u, i n, i n, n c o)$.

\subsubsection{Subproblem $A(i, j, u, v, e x, e x, n c o)$}

Refer to Figure 9. In this case both $r_{u}$ and $r_{v}$ are excluded from $R_{A}$ and the solution should not connect $b_{i}$ and $b_{j}$. Let $r_{t}$ be the topmost red point in $R_{A} . r_{t}$ must be connected to at least one blue point in the final solution. Let $b_{k}$ be the leftmost of those blue points, where $i \leq k \leq j$. We consider two cases: the case where $k \in\{i, j\}$ and the case where $k \in\{i+1, \ldots, j-1\}$. We then take the one that minimizes $A(i, j, u, v$, ex, ex, nco $)$. In the case where $k \in\{i, j\}$, if $k=i$ then

$$
A(i, j, u, v, \text { ex }, \text { ex }, n c o)=\left|r_{t} b_{i}\right|+A(i, i, u, t, \text { ex }, \text { ex }, \text { con })+A(i, j, t, v, \text { in }, \text { ex }, \text { nco })
$$




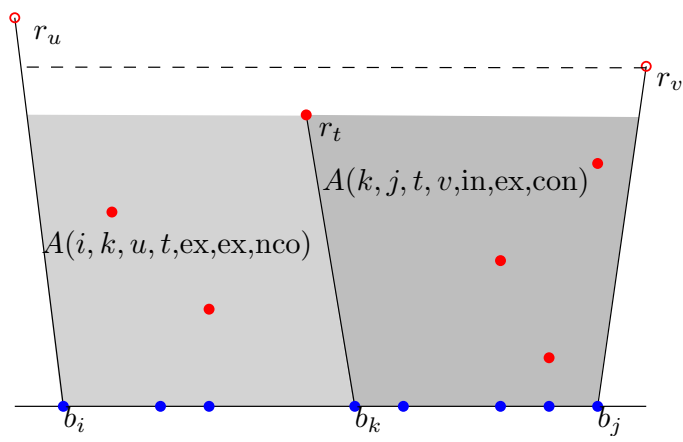

Figure 9: Illustration for solving $A(i, j, u, v$, ex, ex, nco)

and if $k=j$ then

$$
A(i, j, u, v, \text { ex }, \text { ex }, n c o)=\left|r_{t} b_{j}\right|+A(i, j, u, t, \text { ex }, \text { ex }, n c o)+A(j, j, t, v, i n, \text { ex }, \text { con })
$$

In the case where $k \in\{i+1, \ldots, j-1\}$, we have two subproblems. In order to not connect $b_{i}$ to $b_{j}$, only one of the two subproblems should connect $b_{k}$ to either $b_{i}$ or $b_{j}$. This give us two cases. We take the minimum of them. Figure 9 gives an example of solving $A\left(i, j, u, v\right.$, ex, ex, nco) in case 2 . In this example we want to connect $b_{k}$ to $b_{j}$ by a path, but we do not want to connect $b_{i}$ to $b_{k}$ by a path. Thus, we obtain the following:

$$
\begin{aligned}
A(i, j, u, v, \text { ex }, \text { ex }, \text { nco })= & \min \{A(i, k, u, t, \text { ex }, \text { ex }, \text { con })+A(k, j, t, v, \text { in }, \text { ex }, n c o), \\
& A(i, k, u, t, \text { ex }, \text { ex }, \text { nco })+A(k, j, t, v, i n, e x, \text { con })\}+\left|r_{t} b_{k}\right|
\end{aligned}
$$

We try all possible values for $k(i \leq k \leq j)$ and take one that minimizes $A(i, j, u, v$, ex, ex,$n c o)$.

\subsubsection{Subproblem $A(i, j, u, v, i n, e x, n c o)$}

Refer to Figure 10 The solution to $A(i, j, u, v, i n, e x, n c o)$ should contain the edge $r_{u} b_{i}$ and should not connect $b_{i}$ to $b_{j}$. We consider two cases. In the first case, $r_{u}$ is not 


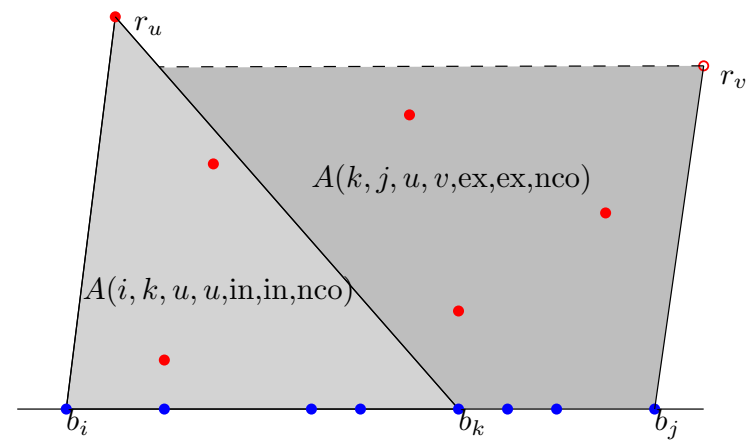

Figure 10: Illustration for solving $A(i, j, u, v$, in, ex, nco)

adjacent to any blue point other than $b_{i}$. In the second case, $r_{u}$ is adjacent to at least one blue point other than $b_{i}$. We then take the case that minimizes $A(i, j, u, v, i n, e x, n c o)$.

In the case where $r_{u}$ is not adjacent to any blue point other than $b_{i}$ we get:

$$
A(i, j, u, v, i n, e x, n c o)=A(i, j, u, v, e x, e x, n c o)
$$

In the case where $r_{u}$ is adjacent to at least one blue point other than $b_{i}$, we let $b_{k}$ be the rightmost blue point adjacent to $r_{u}$, where $i+1 \leq k \leq j-1$. See Figure 10 for an example. Note that in order to maintain disconnectedness, $k$ cannot be equal to $j$. The edge $r_{u} b_{k}$ splits the problem into two independent subproblems. In the left subproblem, $b_{k}$ is connected to $b_{i}$ via $r_{u}$ hence the solution to the right subproblem should be disconnected. Therefore we obtain:

$$
\begin{aligned}
A(i, j, u, v, i n, e x, n c o) & =\min \left\{\left|r_{u} b_{k}\right|+A(i, k, u, u, i n, i n, n c o)\right. \\
& +A(k, j, u, v, \text { ex }, \text { ex }, \text { nco }): i+1 \leq k \leq j-1\}
\end{aligned}
$$

Note that both rule 1 and 2 are satisfied here. We iterate over all $j-i-1$ possible values of $k(i+1 \leq k \leq j-1)$ and pick the one that minimizes $A(i, j, u, v, i n, e x, n c o)$.

Of the eight possible configurations for $A(\cdot)$, only five will ever be called. $A(i, j, u, v, i n, i n, c o n), A(i, j, u, v, e x, i n, c o n)$ and $A(i, j, u, v, e x, i n, n c o)$ will never be 


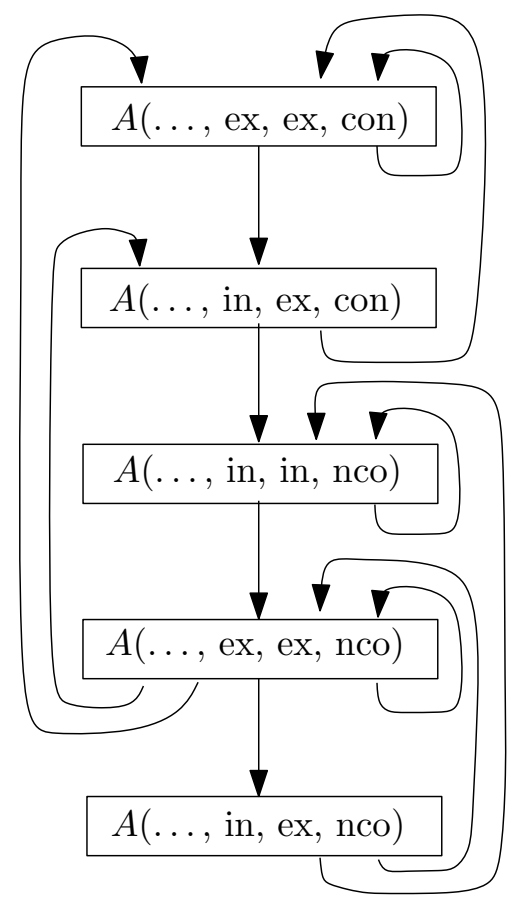

Figure 11: Illustration of the recurrence tree for $A(\cdot)$ 
called. Figure 11 shows what subproblems call each other. Only the five subproblems that can be called are included in this figure. These are the subproblems we have already described. Note that no infinite loop will occur as every time we change to another subproblem we reduce the problem. Note also that the only geometric reasoning we do about the correctness of our algorithm come from the non-crossing constraint. No where do we reason about distances.

\subsubsection{The Top-Level Problem}

In this section we describe the top-level problem and how we are able to use the previously described subproblems to build the solution to the problem at hand: finding the MinBPST. We introduce two fake red points, $r_{0}$ and $r_{m+1} . r_{0}$ is a (fake) red point such that its $y$-coordinate is greater than any red point in $\left\{r_{1}, \ldots r_{m}\right\}$ and it is far enough to the left so that all the red points lie to the right of a line through $r_{0}$ and $b_{1} . r_{m+1}$ is a (fake) red point such that its $y$-coordinate is greater than any red point in $\left\{r_{1}, \ldots r_{m}\right\}$, and it is far enough to the right so that all the red points lie to the left of a line through $r_{m+1}$ and $b_{n}$. We maintain a table where $A\left[i, j, u, v, u^{\prime}, v^{\prime}, w\right]$ is the weight of the MinBPST found by $A\left(i, j, u, v, u^{\prime}, v^{\prime}, w\right)$. Then the weight of the MinBPST we desire to find can be found in the table entry $A[1, n, 0, m+1$, ex, ex, con $]$. We are able to find the MinBPST itself by maintaining the $k$ value at each level that gives the MinBPST for that level and backtracking through the table.

\subsubsection{Base Cases}

We now describe the base cases for the algorithm.

Base Case 1: $A(i, j, u, v$, ex, ex, con $)$ :

We have two subcases: $i=j$ and $i \neq j$. 


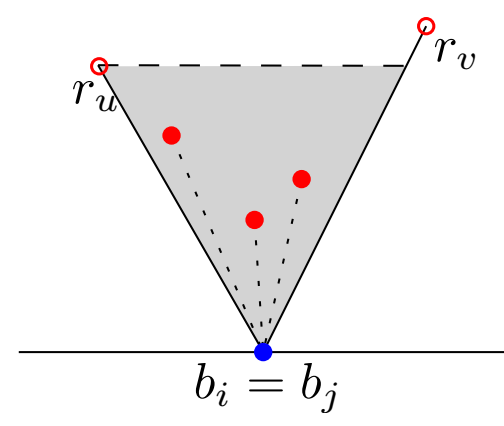

(a)

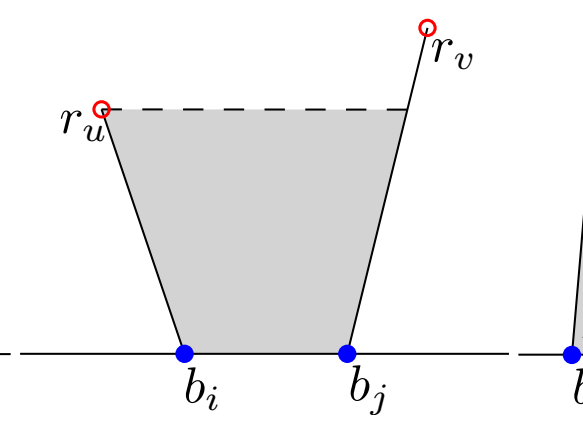

(b)

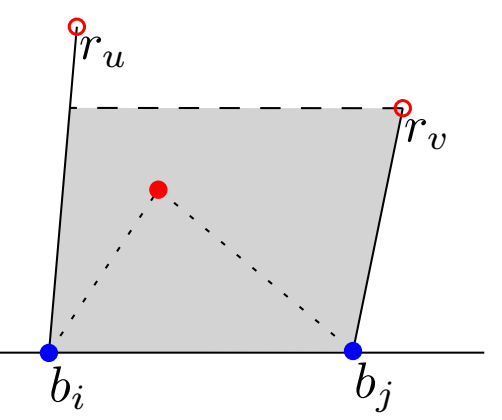

(c)

Figure 12: Illustration of Base Case 1

- subcase $i=j$ :

In the case when $i=j$, we connect all red points in $R_{A}$ to $b_{i}$ (which is equal to $b_{j}$ ). We add the length of each of these edges together and return that value. See Figure 12 (a).

- subcase $i \neq j$ :

When $i \neq j$ we have two cases: there are no points in $R_{A}$ or there is exactly one point in $R_{A}$. Note that since this is a base case there can be at most one red point in $R_{A}$. In the case where $R_{A}$ is empty (Figure $12(\mathrm{~b})$ ), we return $-\infty$. In the case where there is exactly one point in $R_{A}$ (Figure 12(c)), we connect that red point to both $b_{i}$ and $b_{j}$. We add these two edge lengths together and return that value.

Base Case 2: $A(i, j, u, v, i n$, ex, con $)$

We have two subcases: $i=j$ and $i \neq j$.

- subcase $i=j$ : When $i=j$ we connect all of the red points in $R_{A}$, excluding $r_{u}$ which is already connected to $b_{i}$, to $b_{i}$, which is the same point as $b_{j}$ (see Figure 13(a)). We find the total sum of these edges and return that value. Note that $\left|r_{u} b_{i}\right|$ is not included in this sum total by rule 1 . 


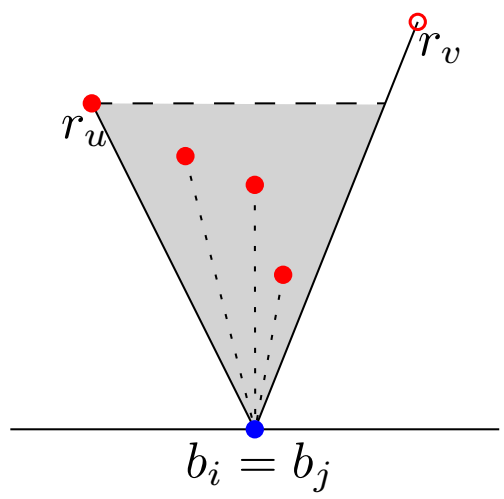

(a)

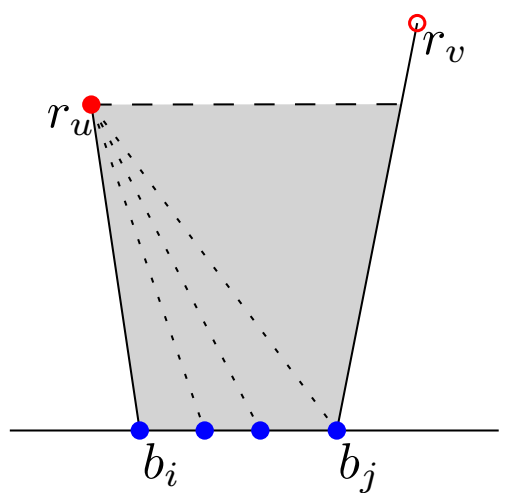

(b)

Figure 13: Illustration of Base Case 2

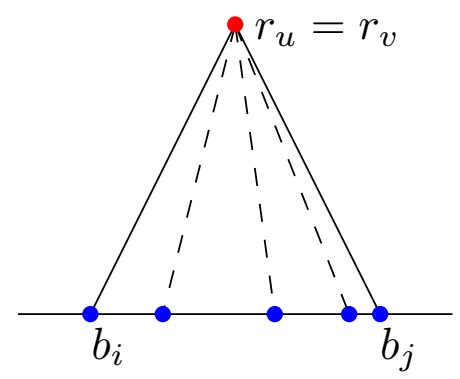

Figure 14: Illustration of Base Case 3

- subcase $i \neq j$ : In this case, the only point in $R_{A}$ is $r_{u}$. We connect $r_{u}$ to each blue point $b_{k}$ where $i+1 \leq k \leq j$ (see Figure 13(b)). We take the sum total of all of the added edge lengths and return that value. $\left|r_{u} b_{i}\right|$ is not included in the summation because of rule 1 .

Base Case 3: $A(i, j, u, v$, in, in, $n c o)$

By rule $2, u=v$ and consequently $r_{u}=r_{\nu}$. For each blue point $b_{k}$ where $i+1 \leq k \leq j-1$, we add an edge from $b_{k}$ to $r_{u}$. We take the sum total of the new edges added and return this value. See Figure 14. 
Base Case 4: $A(i, j, u, v$, ex, ex, nco $)$

In this case there are no points in $R_{A}$ because $r_{u}$ and $r_{\nu}$ are excluded and we are in a base case. $b_{i}$ and $b_{j}$ cannot be equal since they are already the same spanning tree, and thus connected. Their equivalence would create an additional connection between $b_{i}$ and $b_{j}$ and therefore create a cycle in the resulting tree. We have two subcases: $b_{j}=b_{i+1}$ and $b_{j} \neq b_{i+1}$.

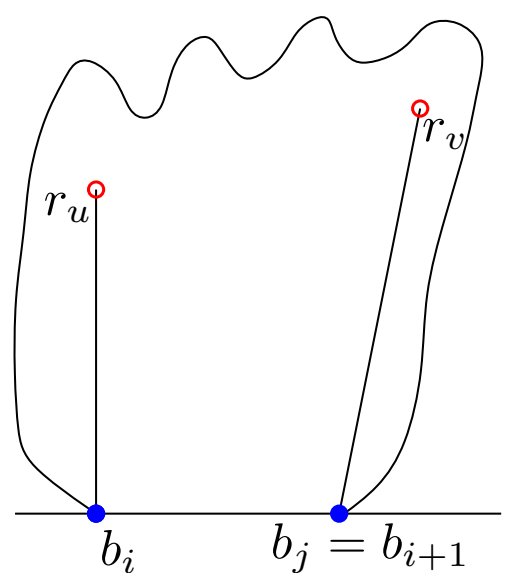

(a)

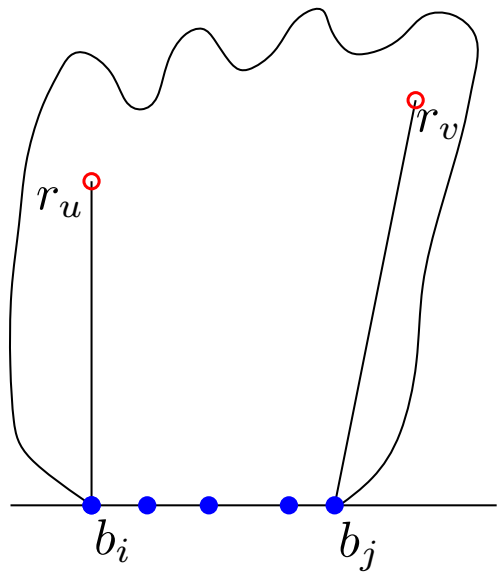

(b)

Figure 15: Illustration of Base Case 4

- Subcase $b_{j}=b_{i+1}$ : In this case there are no blue points between $b_{i}$ and $b_{j}$. Thus, there are no points that need to be connected and hence we return the value 0 . See Figure 15(a).

- Subcase $b_{j} \neq b_{i+1}$ : In this case there is at least one blue point between $b_{i}$ and $b_{j}$. See Figure 15(b). Since there are no red points in $R_{A}$, there is no way of connecting the blue points between $b_{i}$ and $b_{j}$ to the tree, therefore we return $-\infty$.

Base Case 5: $A(i, j, u, v, i n$, ex, nco $)$

In this case the only red point in $R_{A}$ is $r_{u}$ since $r_{v}$ is excluded and we are in a base case. 
$b_{i}$ and $b_{j}$ are already in the same tree so our solution should not connect them again as that would result in a cycle. We have two cases: $b_{j}=b_{i+1}$ and $b_{j} \neq b_{i+1}$.

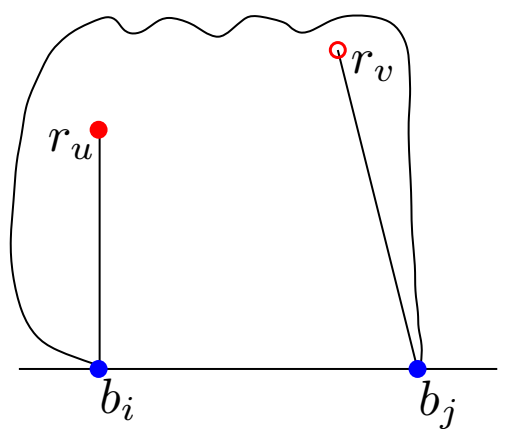

(a)

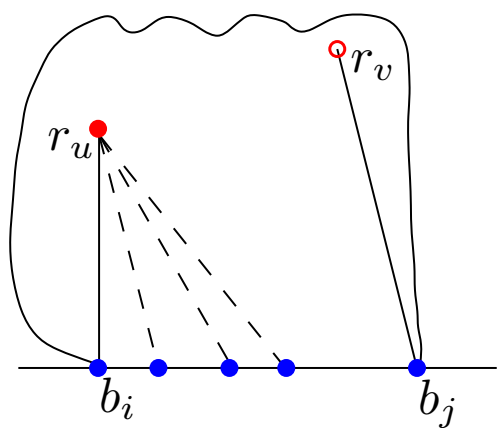

(b)

Figure 16: Illustration of Base Case 5

- Subcase $b_{j}=b_{i+1}$ : In this case there are no blue points between $b_{i}$ and $b_{j}$. Thus, there are no points that need to be connected and hence we return the value 0 . See Figure 16(a).

- Subcase $b_{j} \neq b_{i+1}$ : In this case there is at least one blue point between $b_{i}$ and $b_{j}$. See Figure 16(b). For every blue point $b_{k}$, where $i+1 \leq k \leq j-1$ we connect $b_{k}$ to the red point $r_{u}$. We take the sum total of all of these new edges added and return that value.

To get a feel for how the algorithm can be implemented, see Algorithm 1. Note that for simplicity, we have not included specific base cases in our pseudocode.

\subsubsection{Running Time Analysis}

We now turn our attention to the running time of the algorithm. The indices $i$ and $j$ iterate over all possible $n$ blue points and the indices $u$ and $v$ iterate over all $m$ red points. Therefore we have $O\left(n^{2} m^{2}\right)$ subproblems and hence the table $A$ has $O\left(n^{2} m^{2}\right)$ entries. 


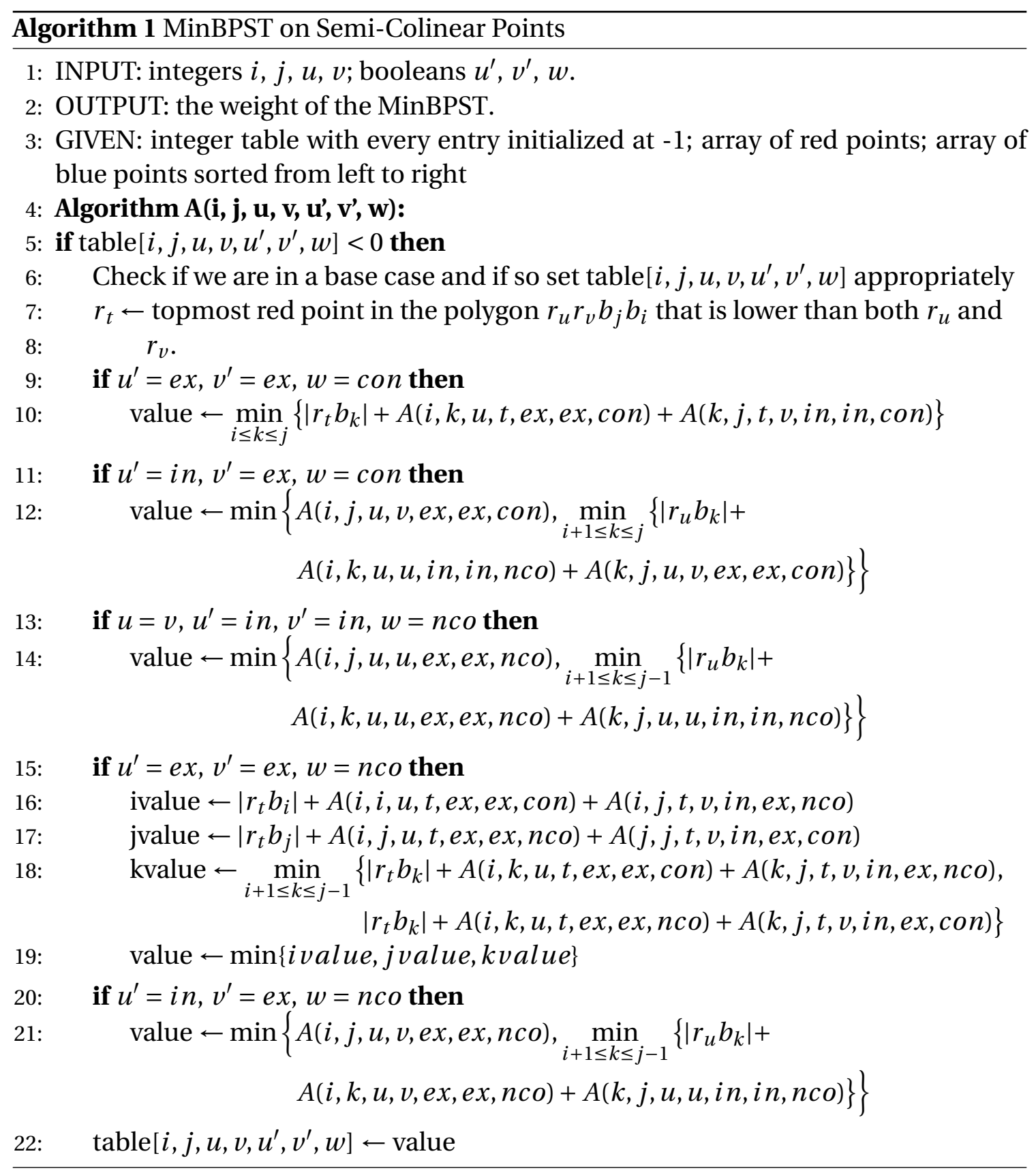


We can find the red points associated with each subproblem and the topmost red point in $O(m)$ time. Thus, the time for preprocessing takes $O\left(n^{2} m^{3}\right)$. During the execution of the algorithm, the index $k$ iterates over $O(n)$ blue points. Hence after the preprocessing, it takes $O\left(n^{3} m^{2}\right)$ to solve the subproblems. Therefore, overall the algorithm takes $O\left(n^{2} m^{3}+n^{3} m^{2}\right)$ time to run.

We now reduce the running time to $O\left(n^{3} \mathrm{~m}^{2}\right)$ by showing how to efficiently compute the topmost red point in $O\left(n^{2} m^{2}\right)$ preprocessing time. When we solve a subproblem, only the topmost red point in that subproblem is actually necessary. Furthermore, we only need to find the topmost red point in subproblems of the form $A(\cdot, \cdot, \cdot, \cdot$, ex, ex, $\cdot)$.

For the subproblem $A(i, j, u, v$, ex, ex, $w)$, the topmost red point is uniquely defined by $i, j, u$, and $v$. The topmost red point does not in any way depend on the value of $w$. We will assume, without loss of generality, that the blue points lie on the $x$-axis and the red points lie above the $x$-axis. For simplicity in our explanation, we assume that no two red points have the same $y$-coordinate, but this is unnecessary for the running of the algorithm. Let $R=\left\{r_{0}, r_{1}, \ldots, r_{m}, r_{m+1}\right\}$ be the set of red points, including the two fake red points, $r_{0}$ and $r_{m+1}$, used in the top level problem. Let $b_{1}, b_{2}, \ldots, b_{n}$ be the blue points ordered from left to right. For each $i$ where $1 \leq i \leq n$, let $L_{i}^{c}$ be the list of red points sorted in clockwise order around the blue point $b_{i}$, starting at the $x$-axis. Similarly, let $L_{i}^{c c}$ be the list of red points sorted in counter-clockwise order around the blue point $b_{i}$, starting at the $x$-axis.

We maintain a table $C$ where $C[i, j, u, v]$ is the entry that stores the topmost red point for the subproblem $A(i, j, u, v$, ex, ex, $w)$, where $1 \leq i, j \leq n$ and $0 \leq u, v, \leq m+1$. Recall that the input red point set for the subproblem $A(i, j, u, v, e x, e x, w)$ is the red points that lie to the right of a line through $b_{i}$ and $r_{u}$, to the left of a line through $b_{j}$ and $r_{\nu}$ and below both $r_{u}$ and $r_{\nu}$.

For each pair of blue points $(i, j)$, where $1 \leq i, j \leq n$, we fill the entries $C[i, j, \cdot, \cdot]$ in two phases. In the first phase we fill the entries of $C$ related to the cases when $r_{u}$ 
is higher than $r_{v}$ and in the second phase we fill the entries of $C$ related to the cases when $r_{v}$ is higher than $r_{u}$. We will describe the first phase. The second phase is similar. We iterate over all possible values for $v(0 \leq v \leq m+1)$, and compute the topmost red point for each red point $r_{u}$ where $0 \leq u \leq m+1$ and $r_{u}$ is above $r_{v}$. Let $r_{t}$ be the current topmost red point that lies to the left of a line through $r_{\nu}$ and below $r_{\nu}$. We traverse the points of $L_{i}^{c c}$ in order. Let $r_{u}$ be the current point. We have two cases:

1) $r_{u}$ is above $r_{\nu}$. Set $C[i, j, u, v]=r_{t}$, then proceed to the next point in $L_{i}^{c c}$.

2) $r_{u}$ is below $r_{v}$. If $r_{u}$ lies to the left of the line through $r_{v}$ and $b_{j}$ and is higher than the $r_{t}$, let $r_{t}=r_{u}$. Then proceed to the next point in $L_{i}^{c c}$.

This concludes the first phase. In the second phase we iterate over all possible values for $u(0 \leq v \leq m+1)$, and compute the topmost red point for each red point $r_{v}$ where $0 \leq v \leq m+1$ and $r_{v}$ is above $r_{u}$. We then traverse over $L_{j}^{c}$ to fill in the entries of $T$ when $b_{v}$ is above $b_{u}$.

We now show that the total running time for the algorithm is $O\left(n^{3} m^{2}\right)$. Creating the lists $L_{i}^{c}$ and $L_{i}^{c c}$ for each blue point $i$ where $1 \leq i \leq n$ takes $O(n m \log m)$ time. It takes us $O\left(n^{3} m^{2}\right)$ to fill the table $T$. Therefore the total running time for our algorithm is $O\left(n^{3} m^{2}\right)$.

Theorem 2.2.1. Let $B$ be a set of $n$ blue points that lie on a line and let $R$ be a set of $m$ red points that lie on one side of the line. A MinBPST of the point set $B \cup R$ can be computed in $O\left(n^{3} m^{2}\right)$ time.

\subsection{Extensions of the Algorithm}

The algorithm for finding the MinBPST can easily be modified to find a maximum bichromatic plane spanning tree, a minimum bottleneck bichromatic plane spanning tree or the maximum bottleneck bichromatic plane spanning tree on semi-collinear points in $O\left(n^{3} m^{2}\right)$ time. 
Maximum Bichromatic Plane Spanning Tree: A maximum bichromatic plane spanning tree is a bichromatic plane spanning tree that has maximum weight. In order to adapt our algorithm to find the maximum plane bichromatic spanning tree we change all the minimums to maximums in Algorithm 1. We change our base cases so that they return the maximum value of the bichromatic plane spanning trees found. If a base case is infeasible we return $-\infty$.

Minimum Bottleneck Bichromatic Plane Spanning Tree: A minimum bottleneck bichromatic plane spanning tree is a bichromatic plane spanning tree whose longest edge is minimal. In our base cases we return the length of the longest edge. This is the value we desire to minimize. We take the minimum of the longest edge of every possible tree. For example line 10 in Algorithm 1 becomes:

$$
\text { value } \leftarrow \min \left\{\max _{i \leq k \leq j}\left\{\left|r_{t} b_{k}\right|, A(i, k, u, t, e x, e x, c o n), A(k, j, t, v, i n, i n, c o n)\right\}\right\}
$$

Maximum Bichromatic Plane Spanning Tree: A maximum bottleneck bichromatic plane spanning tree is a bichromatic plane spanning tree whose shortest edge is maximal. In our base cases we return the length of the shortest edge. This is the value we desire to maximize. We take the maximum of the shortest edge of every possible tree. For example line 10 in Algorithm 1 becomes:

$$
\text { value } \leftarrow \max \left\{\min _{i \leq k \leq j}\left\{\left|r_{t} b_{k}\right|, A(i, k, u, t, e x, e x, \text { con }), A(k, j, t, v, \text { in }, \text { in }, \text { con })\right\}\right\}
$$

\subsection{Implementation of the Algorithm}

We implemented the algorithm for finding the MinBPST in Java. The algorithm can easily be altered to find the maximum bichromatic plane spanning tree, the minimum bottleneck bichromatic plane spanning tree and the maximum bottleneck bichromatic plane spanning tree, hence we also implemented these versions of the algorithm. 
While using the implementation, a user is able to input the desired number of red and blue points. The user must input the number of points desired before they are able to do anything else. The user is then able to either select the points they wish to use or randomly generate the points.

To select the points the user selects "Select Points" and then simply uses their mouse to click the desired location of the points. Since the blue points must lie on the line, only the $x$-coordinate is considered when the user selects the blue points. For example, if the user is selecting a blue point by clicking near the top of the designated area for selecting points, with $x=600$, the blue point will appear on the bottom line with $x=600$. We ensure that no three points, where two of the points are red and one of the points is blue, are collinear, by simply off-setting the selected location of the point by a pixel.

To randomize the points the user simply selects "Randomly Generate Points" and then presses "Go". Our implementation uses a pseudo-random number generator to select the points. Our implementation also ensures that the randomization process does not create points that overlap and ensures that no three points, where two of the points are red and one of the points is blue, are collinear. To do so, a new pseudorandom point is generated until those restrictions are met.

After all the points have been selected, either by user input, or by randomization, the user is able to select the kind of bichromatic plane spanning tree they would like to produce. The selections are: "Minimum", "Maximum", "Minimum Bottleneck" and "Maximum Bottleneck".

The user is also able to clear the current point set and possible bichromatic plane spanning tree by pressing the "Clear" button.

Unfortunately, due to the deep recursive nature of the algorithm, there are limitations of the implementation. A stack overflow error is likely to occur when the number of points is too large. There is no specific upper bound for the number of points because the depth of the recursion, and hence the likelihood of a stack overflow error, is 
also dependent on the positioning of the points.

Figures 17, 18, 19 and 20 show example case of the implementation. In each example we randomly generated 7 red points and 7 blue points. Figure 17 finds the MinBPST. Figure 18 finds the maximum bichromatic plane spanning tree. Figure 19 finds the minimum bottleneck bichromatic plane spanning tree. Figure 20 finds the maximum bottleneck bichromatic plane spanning tree. 


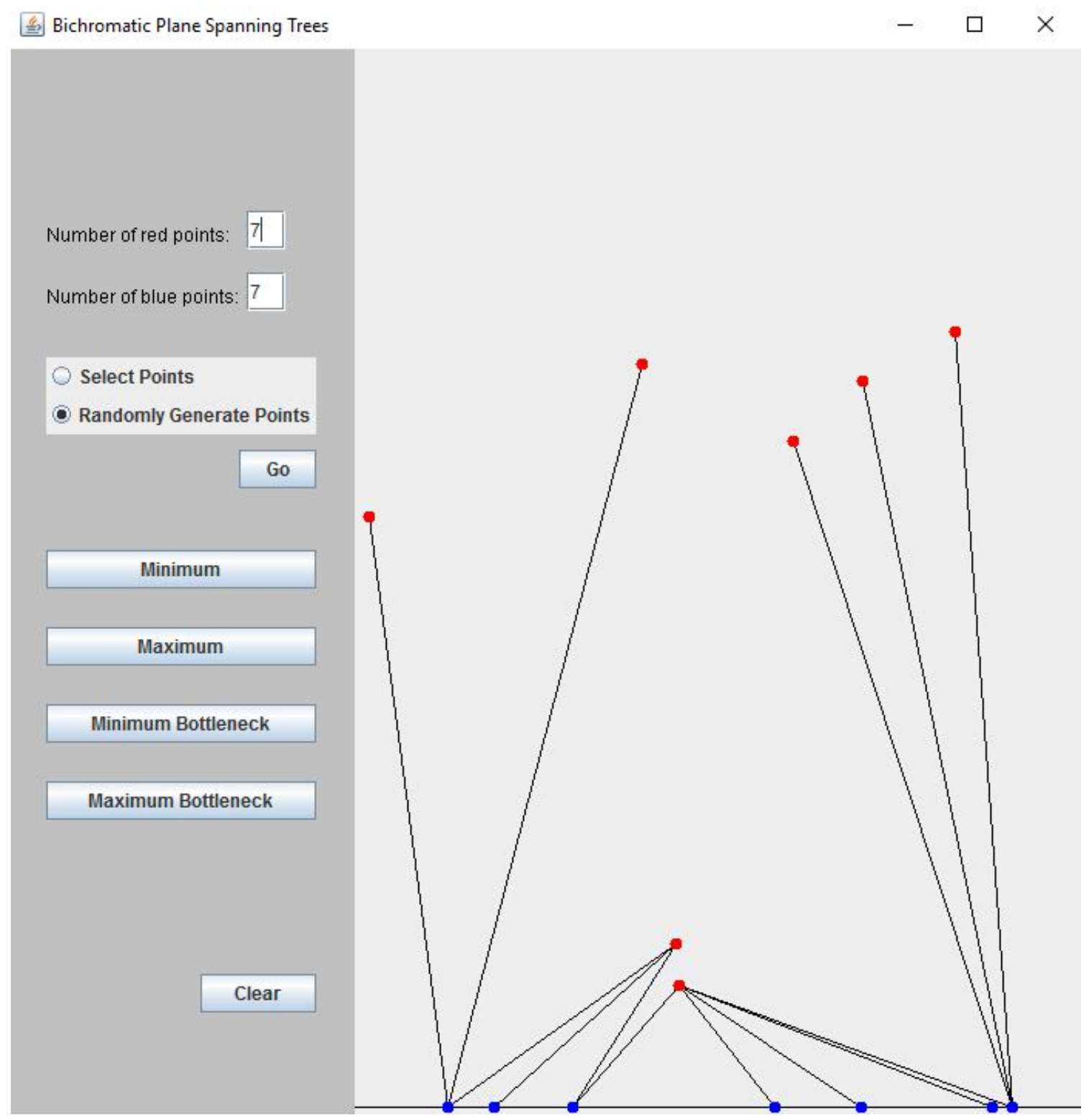

Figure 17: An example of the implementation with a generated minimum bichromatic plane spanning tree 
Bichromatic Plane Spanning Trees

$-\square \times$

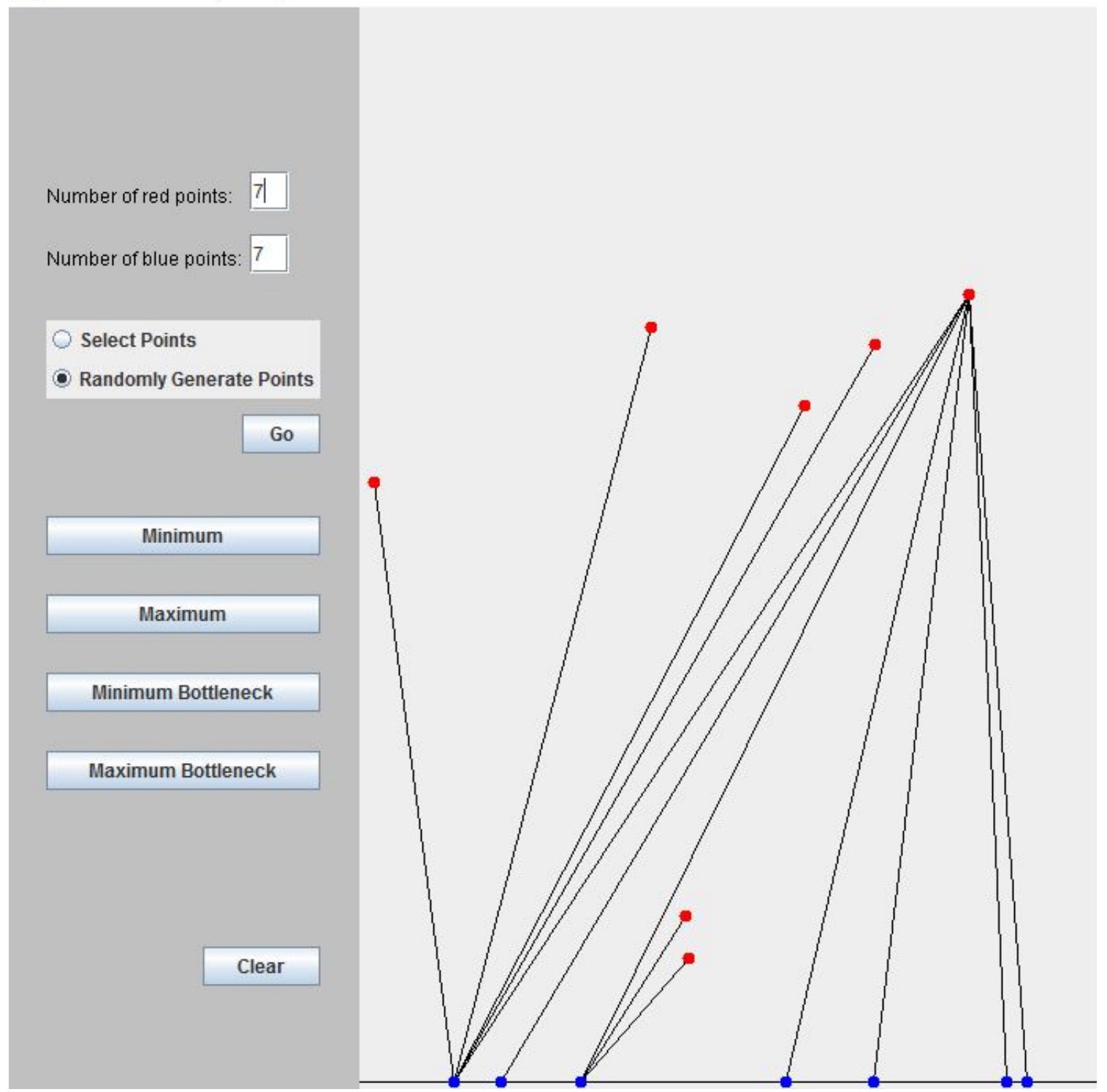

Figure 18: An example of the implementation with a generated maximum bichromatic plane spanning tree 
Bichromatic Plane Spanning Trees
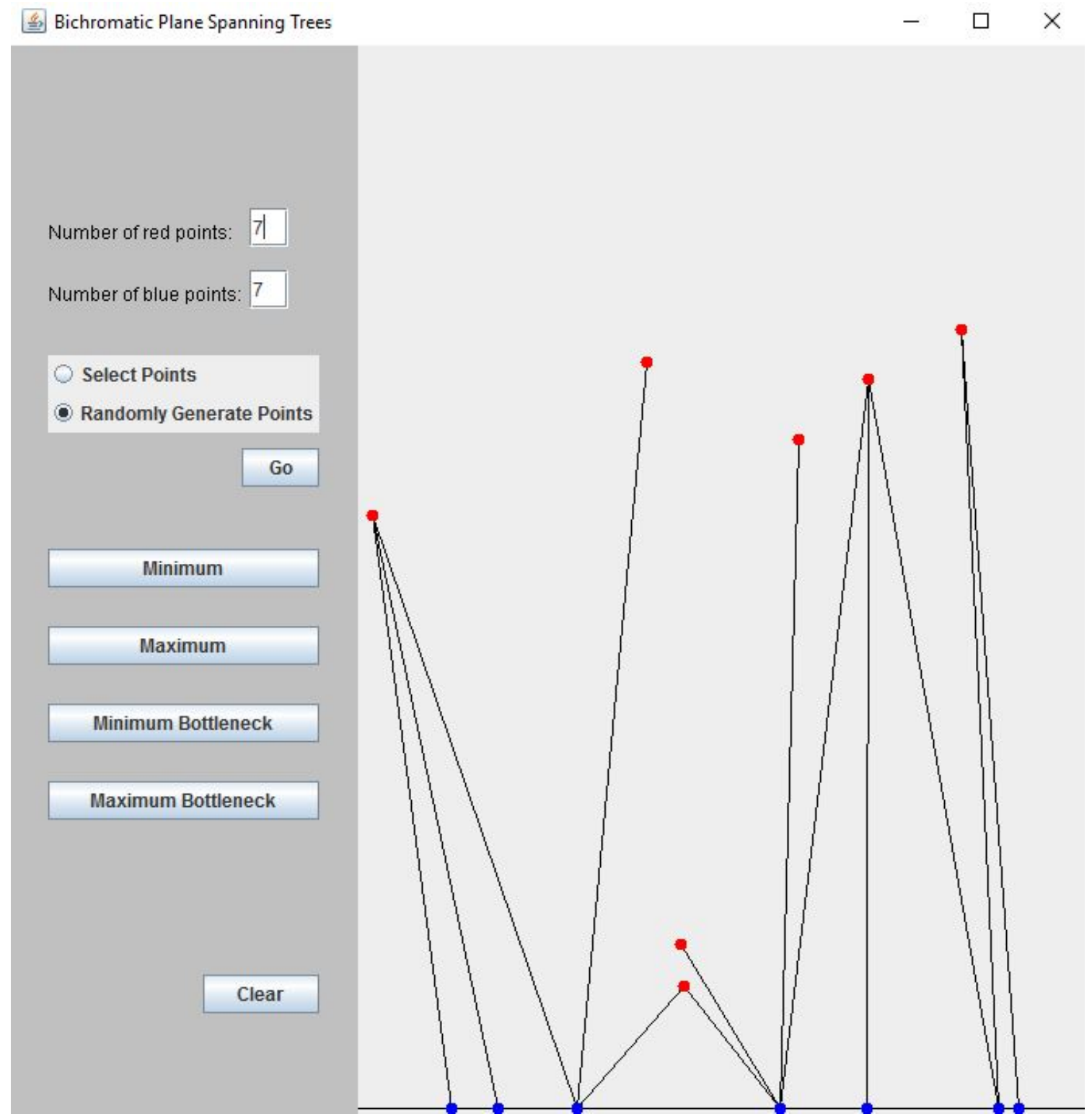

Figure 19: An example of the implementation with a generated minimum bottleneck bichromatic plane spanning tree 


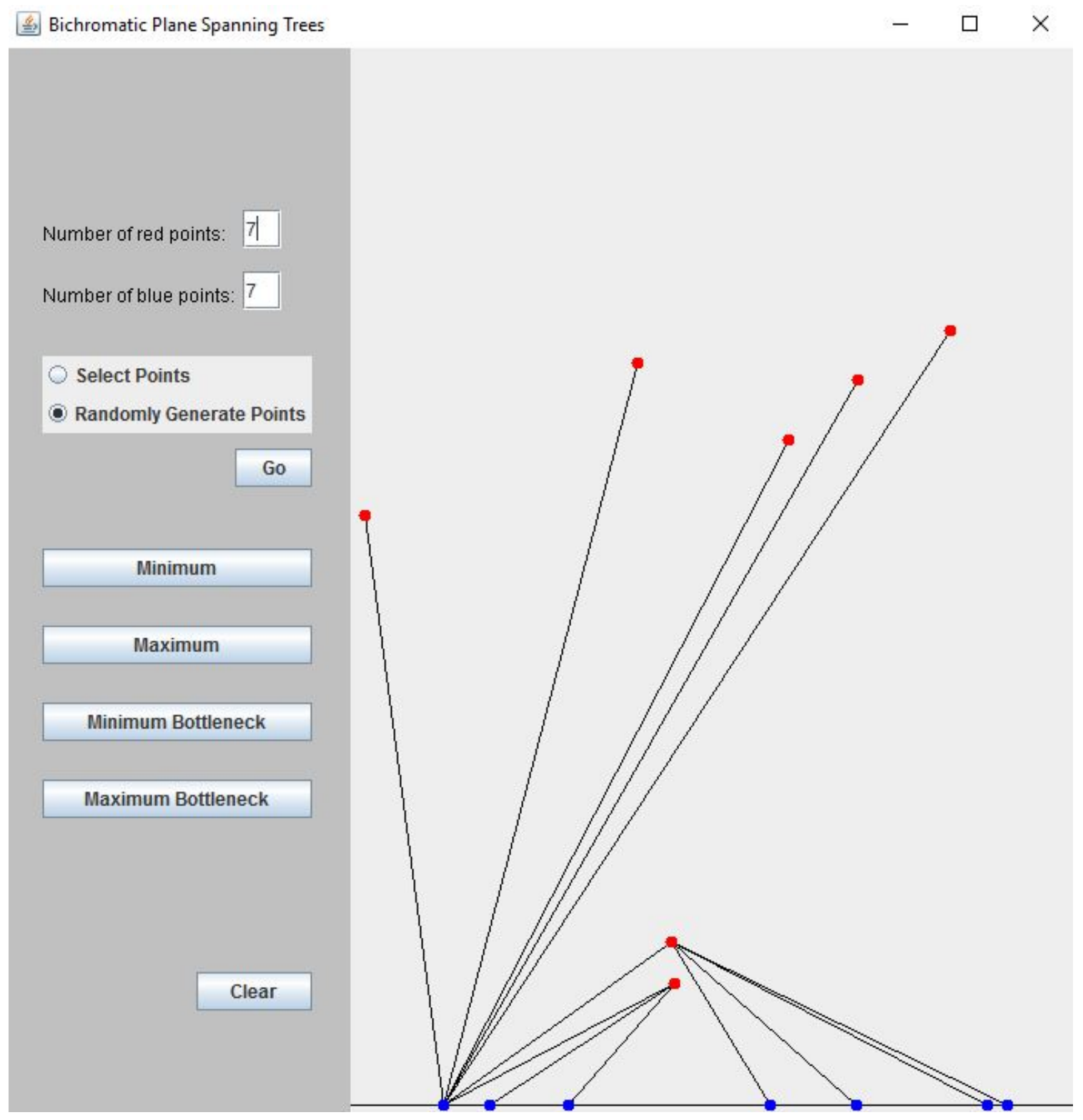

Figure 20: An example of the implementation with a generated maximum bottleneck bichromatic plane spanning tree 


\subsection{Conclusion}

In this chapter we looked at how to find the MinBPST $T^{*}$ when the blue points lie on a line and the red points lie on one side of the line. First we showed using a counterexample that a greedy algorithm will not necessarily find $T^{*}$. Next we described a dynamic programming algorithm that finds $T^{*}$ in $\mathrm{O}\left(|B|^{3}|R|^{2}\right)$ time. Finally, we described a Java implementation of the algorithm with the ability to additionally find the maximum, minimum bottleneck and maximum bottleneck plane spanning trees. 


\section{Chapter 3}

\section{Properties of MinBPST on Semi-Collinear Points}

Suppose we have a set $B$ of blue points that lie on a line and a set $R$ of red points that lie on one side of the line. Without loss of generality, suppose the line is horizontal and the red points lie above the line. In this chapter we will always assume that there is a red point $r$ that is adjacent to two blue points, $b_{i}$ and $b_{j}$. We consider the MinBPST, $T^{*}$, on such a point set. $T^{*}$ has special properties. In this chapter we describe the following properties: the existence of split points, that if $\max \left\{\angle r b_{i} b_{j}, \angle r b_{j} b_{i}\right\} \geq 90^{\circ}$, then there is no red point in the triangle $\Delta r b_{i} b_{j}$ and that in certain circumstances no edge crosses the vertical line through the highest red point. We also prove why these properties must hold. We assume no three points, where two of the points are red and one of the points is blue, are collinear. We hope that these properties can be used by future researchers to create an algorithm that is more efficient than the one presented in chapter 2.

\subsection{Preliminaries}

We will now define several terms that are used throughout the chapter.

Given a plane tree, $T$, and three points, $r, b$, and $c$, a $\bar{r}$-path $(b, c)$ is a path that connects $b$ to $c$ without using the point $r$. We say that $b$ and $c$ are $\bar{r}$-connected if there exists a $\bar{r}-\operatorname{path}(b, c)$. 
Given a point $b$ that lies on a line $\ell$, we define the rightmost edge with endpoint $b$ to be the edge of $T$ with endpoint $b$ that has the smallest angle counter-clockwise from $\ell$. We define the leftmost edge with endpoint $b$ to be the edge of $T$ with endpoint $b$ that has the smallest angle clockwise from $\ell$. Figure 21 gives a visualization of a rightmost edge with endpoint $b$ and a leftmost edge with endpoint $b$.

We say a point $b$ is visible from another point $c$ if the edge $b c$ does not cross any other edges of $T$. We call an edge $b c$ free if $b$ is visible from $c$ and vice versa.

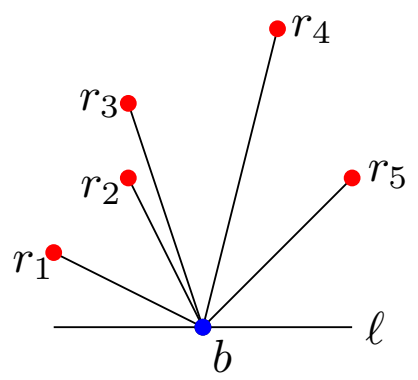

Figure 21: Illustration of the definition of rightmost and leftmost edges. Here, $r_{1} b$ is the leftmost edge with endpoint $b$ whereas $r_{5} b$ is the rightmost edge with endpoint $b$.

\subsection{Split Points}

The MinBPST, $T^{*}$, for a set of semi-collinear points contains split points. Suppose there is a red point $r \in R$ and $r$ is connected by an edge to two different blue points. Split points are two consecutive points inclusively between these blue points (they could possibly include one or both of the two original points), such that the removal of the two edges connected to $r$ creates two bichromatic plane trees where the blue points contained in the first tree are all consecutive and the blue points in the other tree are all consecutive. The split points are the rightmost blue point of the first tree and the leftmost point of the second tree. Figure 22 gives an example of split points. Note that 
this figure depicts a spanning tree that is not the MinBPST, but is able to illustrate the idea of split points well. We define split points formally and prove their existence under a specific hypothesis in the proof of the following lemma.

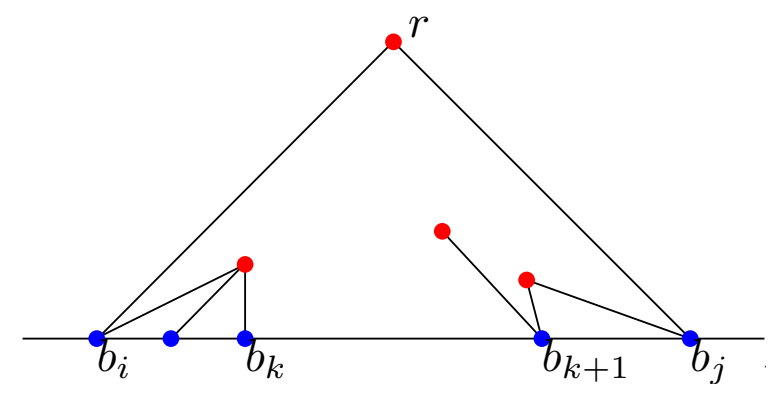

Figure 22: Illustration of split points.

Lemma 3.2.1 (Existence of split points). Suppose we are given a set $B$ of $n$ blue points on a horizontal line $\ell$, ordered from left to right as $b_{1}, \ldots, b_{n}$ and a set $R$ of $m$ red points that lie above $\ell$. Let $T^{*}$ be a MinBPST of the point set $B \cup R$ and $r \in R$ be a red point. Suppose $r b_{i}$ and $r b_{j}$ are edges in $T^{*}$, but $r b_{p}$ is not an edge in $T^{*}$ for all $i<p<j$. Then, in $T^{*}$ there exist two consecutive split points $b_{k}$ and $b_{k+1}$ such that $b_{i+1}, \ldots, b_{k}$ are $\bar{r}$-connected to $b_{i}$, but not $\bar{r}$-connected to $b_{j}$ and $b_{k+1}, \ldots, b_{j-1}$ are $\bar{r}$-connected to $b_{j}$, but not $\bar{r}$-connected to $b_{i}$.

Proof. By way of contradiction, suppose no split points exist. Let $T^{\prime}=T^{*}-\{r\}$. We consider two cases. In the first case, there exists a path from $b_{i}$ to $b_{j}$ in $T^{\prime}$. This causes a cycle in $T^{*}$ and contradicts the fact that $T^{*}$ is a tree. In second case, there does not exist a path from $b_{i}$ to $b_{j}$ in $T^{\prime}$ and $T^{\prime}$ is composed of two disjoint connected components. Since there are no split points, there must exist at least two points $b_{s}$ and $b_{q}$ where $q>s$ such that there is a path from $b_{s}$ to $b_{j}$ and a path from $b_{q}$ to $b_{i}$. These paths must cross since $b_{s}$ lies between $b_{i}$ and $b_{j}$ and $b_{q}$ lies between $b_{s}$ and $b_{i}$. This contradicts the fact that $T^{\prime}$ is plane and hence $T^{*}$ must contain split points. 


\subsection{Obtuse Triangles}

In $T^{*}$ on a set of semi-collinear points, $\max \left\{\angle r b_{i} b_{j}, \angle r b_{j} b_{i}\right\} \geq 90^{\circ}$, then there is no red point in the triangle $\triangle r b_{i} b_{j}$. Because of this fact, we obtain Lemma 3.3.1. Additionally, we get a corollary that says that if there are any blue points in a triangle $\Delta r b_{i} b_{j}$ where $\max \left\{\angle r b_{i} b_{j}, \angle r b_{j} b_{i}\right\} \geq 90^{\circ}$, they must all be connected to the vertex red point $r$.

Lemma 3.3.1. Suppose we are given a set $B$ of $n$ blue points on a horizontal line $\ell$, ordered from left to right as $b_{1}, \ldots, b_{n}$ and a set $R$ of $m$ red points that lie on one side of $\ell$. Let $T^{*}$ be a MinBPST of the point set $B \cup R$ and $r \in R$ be a red point. If $r b_{i}$ and $r b_{j}$ are edges in $T^{*}$ and $\max \left\{\angle r b_{i} b_{j}, \angle r b_{j} b_{i}\right\}>90^{\circ}$, then there is no red point in the triangle $\triangle r b_{i} b_{j}$. That is to say, if the triangle formed by the edges $r b_{i}$ and $r b_{j}$ and the line segment of $\ell$ between $b_{i}$ and $b_{j}$, is obtuse, it cannot contain any red points.

Proof. By way of contradiction, assume $\triangle r b_{i} b_{j}$ contains at least one red point, where $r b_{i}$ and $r b_{j}$ are edges in $T^{*}$, but $r b_{p}$ is not an edge in $T^{*}$ for all $i<p<j$. Note that it could be the case that there are no blue points between $b_{i}$ and $b_{j}$ (i.e. $b_{j}=b_{i+1}$ ). We consider three cases: no red points are $\bar{r}$-connected to $b_{i}$, no red points are $\bar{r}$-connected to $b_{j}$, and some red points are $\bar{r}$-connected to $b_{i}$ but not $b_{j}$, while some other red points are $\bar{r}$-connected to $b_{j}$, but not $b_{i}$.

Consider the case where no point is $\bar{r}$-connected to $b_{i}$. Then, in order for the tree to be connected, there exists at least one red point that is $\bar{r}$-connected to $b_{j}$. Consider the point $b_{i+1}$. It must be connected to at least one red point and $\bar{r}$-connected to $b_{j}$. Take the leftmost edge with endpoint $b_{i+1}$, call the red endpoint of the edge $r_{i}$. Connect $r_{i}$ and $b_{i}$. Adding the edge $r_{i} b_{i}$ will not cause a crossing, otherwise we contradict the fact that $r_{i} b_{i+1}$ is the leftmost edge with endpoint $b_{i+1}$. Since $r_{i} b_{i}$ lies inside an obtuse triangle, $\left|r_{i} b_{i}\right|<\max \left\{\left|r b_{i}\right|,\left|r b_{j}\right|\right\}$. Thus, we can add $r_{i} b_{i}$ to our tree and remove $\max \left\{\left|r b_{i}\right|,\left|r b_{j}\right|\right\}$ creating a MinBPST with lesser weight than $T^{*}$ and contradicting $T^{*}$ 's optimality. Similarly, in the case where no point is $\bar{r}$-connected to $b_{j}$, we can add the 
edge $r_{j} b_{j}$, where $r_{j}$ is the red endpoint of the rightmost edge with endpoint $b_{j-1}$ and remove $\max \left\{\left|r b_{i}\right|,\left|r b_{j}\right|\right\}$, creating a MinBPST with lesser weight than $T^{*}$, contradicting $T^{*}$ 's optimality.

Now consider the case when some red points are $\bar{r}$-connected to $b_{i}$, but not $b_{j}$, while some other red points are $\bar{r}$-connected to $b_{j}$, but not $b_{i}$. We look at the split points $b_{k}$ and $b_{k+1}$, which must exist by Lemma 3.2.1. Consider the rightmost edge with endpoint $b_{k}$ and call the red endpoint of that edge $r^{\prime}$. Consider the leftmost edge with endpoint $b_{k+1}$ and call the red endpoint of that edge $r^{\prime \prime}$. If $r^{\prime}$ is lower than $r^{\prime \prime}$ by $y$-coordinate, then connect $r^{\prime}$ to $b_{k+1}$ and remove $\max \left\{\left|r b_{i}\right|,\left|r b_{j}\right|\right\}$. This creates a new MinBPST that contradicts the optimality of $T^{*}$. Figure 23 gives an illustration of such a case. Similarly, if $r^{\prime \prime}$ is lower than $r^{\prime}$ by $y$-coordinate, then we connect $r^{\prime \prime}$ to $b_{k}$ and remove $\max \left\{\left|r b_{i}\right|,\left|r b_{j}\right|\right\}$, contradicting $T^{*}$ 's optimality. Note that no red points can lie in the region bounded by $r^{\prime} b_{k}, \ell, r^{\prime \prime} b_{k+1}$ and the horizontal line parallel to $\ell$ that goes through the lower of $r^{\prime}$ and $r^{\prime \prime}$. If a red point were to lie inside this region, it would contradict our choice of leftmost and rightmost edges. Thus our addition of $r^{\prime} b_{k+1}$ when $r^{\prime}$ is lower than $r^{\prime \prime}$ and our addition of $r^{\prime \prime} b_{k}$ when $r^{\prime \prime}$ is lower than $r^{\prime}$ do not create a crossing and thus we maintain the fact that the tree is plane.

Corollary 3.3.2. Suppose we are given a set $B$ of $n$ blue points on a horizontal line $\ell$, ordered from left to right as $b_{1}, \ldots, b_{n}$ and a set $R$ of $m$ red points that lie above $\ell$, where $T^{*}$ is a MinBPST of the point set $B \cup R$. Let $r \in R$. Suppose $r b_{i}$ and $r b_{j}$ are edges in $T^{*}$ and $\max \left\{\angle r b_{i} b_{j}, \angle r b_{j} b_{i}\right\} \geq 90^{\circ}$, then $r b_{p}$ is an edge in $T^{*}$ for all $i<p<j$.

Proof. By Lemma 3.3.1, $\triangle r b_{i} b_{j}$ cannot contain any red points. In order for $T^{*}$ to be connected and for no crossings to occur, $r b_{p}$ must be an edge in $T^{*}$ for all $i<p<j$. 


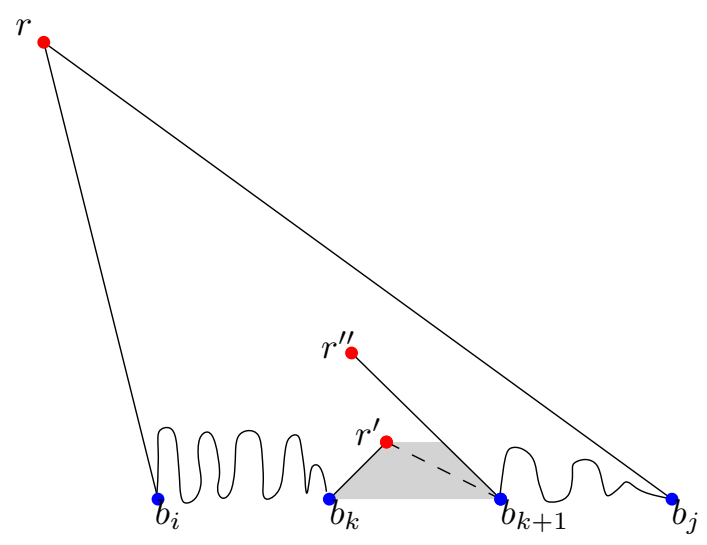

Figure 23: Illustration of proof of Lemma 3.3.1. Here we would add the edge $r^{\prime} b_{k+1}$ and remove the edge $r b_{j}$.

\subsection{Nonexistence of $\ell^{\prime}$-Crossing Edges}

In a MinBPST $T^{*}$, consider a triangle formed by a red point $r \in R$ and two blue points $b_{i}$ and $b_{j}$ with $b_{i}, b_{j} \in B$ where $\max \left\{\angle r b_{i} b_{j}, \angle r b_{j} b_{i}\right\}<90^{\circ}$. Suppose further that $b_{i}$ and $b_{j}$ are not split points. Consider the line $\ell^{\prime}$, that goes through $r$ and is perpendicular to the line that the blue points lie on. We call an edge that crosses this line a $\ell^{\prime}$-crossing edge. In $T^{*}$ no $\ell^{\prime}$-crossing edges can occur. Lemma 3.4.1 and its proof are a formal explanation of the nonexistence of $\ell^{\prime}$-crossing edges.

Lemma 3.4.1. Suppose we are given a set $B$ of $n$ blue points on a horizontal line $\ell$, ordered from left to right as $b_{1}, \ldots, b_{n}$ and a set $R$ of $m$ red points that lie to one side of the line. Let $T^{*}$ be a MinBPST of the point set $B \cup R$ and $r \in R$ be a red point. Draw a line $\ell^{\prime}$ that passes through $r$ and is perpendicular to $\ell$. If $r b_{i}$ and $r b_{j}$ are edges in $T^{*}$, $\max \left\{\angle r b_{i} b_{j}, \angle r b_{j} b_{i}\right\}<90^{\circ}$, and $b_{i}$ and $b_{j}$ are not split points, then no edge of $T^{*}$ crosses the line $\ell^{\prime}$.

Proof. By way of contradiction, suppose there exists an edge $r^{*} b^{*}$ that crosses $\ell^{\prime}$. We have four cases: 1) $b^{*}$ lies on the same side of $\ell^{\prime}$ as $b_{i}$ and $r^{*}$ is $\bar{r}$-connected to $b_{i}$, but 
not $b_{j}$,2) $b^{*}$ lies on the same side of $\ell^{\prime}$ as $b_{i}$ and $r^{*}$ is $\bar{r}$-connected to $b_{j}$, but not $b_{i}, 3$ ) $b^{*}$ lies on the same side of $\ell^{\prime}$ as $b_{j}$ and $r^{*}$ is $\bar{r}$-connected to $b_{j}$, but not $b_{i}$, and 4) $b^{*}$ lies on the same side of $\ell^{\prime}$ as $b_{j}$ and $r^{*}$ is $\bar{r}$-connected to $b_{i}$, but not $b_{j}$. Examples of case 1) and case 2) are given in Figure 24. Case 3) is the symmetric version of case 1) and case 4 ) is the symmetric version of case 2 ), hence we will only prove cases 1) and 2). The proofs of cases 3) and 4) are very similar.

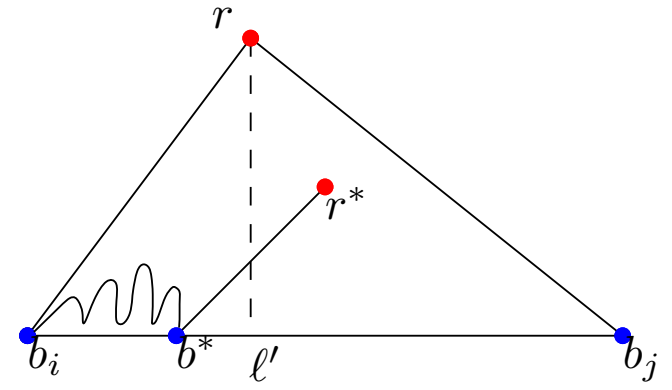

(a)

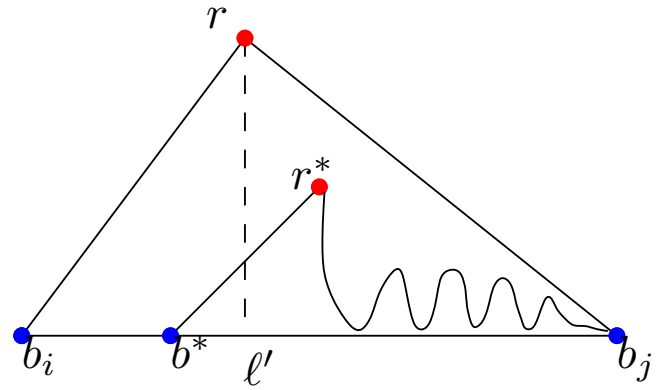

(b)

Figure 24: Illustration of proof of Lemma 3.4.1. (a) Case 1: $r^{*}$ is $\bar{r}$-connected to $b_{i}$, but not $b_{j}$. (b) Case 2: $r^{*}$ is $\bar{r}$-connected to $b_{j}$, but not $b_{i}$.

Consider case 1) where $b^{*}$ lies on the same side of $\ell^{\prime}$ as $b_{i}$ and $r^{*}$ is $\bar{r}$-connected to $b_{i}$, but not $b_{j}$. If more than one crossing edge exists, let $r^{*} b^{*}$ be the edge that crosses $\ell^{\prime}$ with then lowest $y$-coordinate. Try to draw an edge from $r^{*}$ to $b_{j}$. If an edge cannot be added, try to draw an edge from $r^{*}$ to the blue endpoint, $b_{j 1}$, of the edge that prevents the original edge from being drawn. If that edge cannot be drawn, continue the process until an edge can be drawn. At some point this must be the case. Suppose $b_{j k}$ is the blue point that can be connected to $r^{*}$. If $b_{j k}$ lies to the right of $\ell^{\prime}$, then $r^{*} b_{j k}$ lies in the interior of the triangle formed by $\ell^{\prime}, r b_{j}$ and a segment of $\ell$. Since this triangle is a right angle triangle, the longest side of the triangle is $r b_{j}$. Thus, it must be the case that $\left|r^{*} b_{j k}\right|<\left|r b_{j}\right|$. We can add $r^{*} b_{j k}$ to $T^{*}$ and remove $r b_{j}$ from $T^{*}$ to form a MinBPST with lesser weight, contradicting the fact that $T^{*}$ is optimal. If $b_{j k}$ lies to the left of $\ell^{\prime}$, then 
$r^{*} b_{j k}$ lies in the interior of the triangle bounded by $\ell, r^{*} b^{*}$, and the line perpendicular to $\ell$ that goes through $r^{*}$. This is a right angle triangle, with largest edge $r^{*} b^{*}$, hence $\left|r^{*} b_{j k}\right|<\left|r^{*} b^{*}\right|$. Thus, we can add the edge $r^{*} b_{j k}$ and remove $r^{*} b^{*}$, creating a MinBPST with lesser weight than $T^{*}$, contradicting its optimality.

Next, we consider case 2) where $b^{*}$ lies on the same side of $\ell^{\prime}$ as $b_{i}$ and $r^{*}$ is $\bar{r}$ connected to $b_{j}$, but not $b_{i}$. We look at the split points $b^{\prime}$ and $b^{\prime \prime}$ (which must exist by Lemma 3.2.1), where $b^{\prime}$ lies to the right of $b^{\prime \prime}$. Since $b^{*}$ lies on the same side of $\ell^{\prime}$ as $b_{i}$, the split points $b^{\prime}$ and $b^{\prime \prime}$ must also lie on the same side of $\ell^{\prime}$ as $b_{i}$. We now consider three subcases: (a) all edges connected to $b^{\prime}$ and $b^{\prime \prime}$ lie on the same side of $\ell^{\prime}$ as $b_{i}$, (b) at least one edge connected to $b^{\prime \prime}$ crosses $\ell^{\prime}$ and $r^{\prime \prime}$ has a higher $y$-coordinate than $r^{\prime}$, where $r^{\prime}$ is the red endpoint of the rightmost edge with endpoint $b^{\prime}$ and $r^{\prime \prime}$ is red endpoint of the leftmost edge with endpoint $b^{\prime \prime}$ that crosses $\ell^{\prime}$, and (c) is the same as case (b), but $r^{\prime \prime}$ has lower $y$-coordinate than $r^{\prime}$. Note that it cannot be the case that an edge with endpoint $b^{\prime}$ crosses $\ell^{\prime}$, otherwise we are in case 1 .

Consider case 2(a) (Figure 25 (a)). Take the rightmost edge with endpoint $b^{\prime}$ and let its red endpoint be called $r^{\prime}$. Take the leftmost edge with endpoint $b^{\prime \prime}$ and let its red endpoint be called $r^{\prime \prime}$. Then, the region, $\mathscr{R}$, bounded by $r^{\prime} b^{\prime}, \ell, r^{\prime \prime} b^{\prime \prime}$ and the line parallel to $\ell$ that goes through the red endpoint $r^{\prime}$ or $r^{\prime \prime}$ that has lower $y$-coordinate, cannot contain any red points. If $\mathscr{R}$ does contain a red point, it contradicts the fact that our tree is plane or our choice of leftmost and rightmost edges. Suppose $r^{\prime \prime}$ has a lower $y$-coordinate than $r^{\prime}$. Since $\mathscr{R}$ does not contain any red points, it cannot contain any edges, thus we can add the edge $r^{\prime \prime} b^{\prime}$ without causing any crossings and hence maintaining the property that our tree is plane. Since $r^{\prime \prime} b^{\prime}$ lies within the right angle triangle bounded by $r b_{i}, \ell$ and $\ell^{\prime},\left|r^{\prime \prime} b^{\prime}\right|<\left|r b_{i}\right|$, and we can remove the edge $r b_{i}$, creating a MinBPST with weight less than $T^{*}$ and contradicting $T^{*}$ 's optimality. Similarly, if $r^{\prime}$ has a lower $y$-coordinate than $r^{\prime \prime}$, we can create a MinBPST with lesser weight than $T^{*}$ by adding the edge $r^{\prime} b^{\prime \prime}$ and removing $r b_{i}$. 


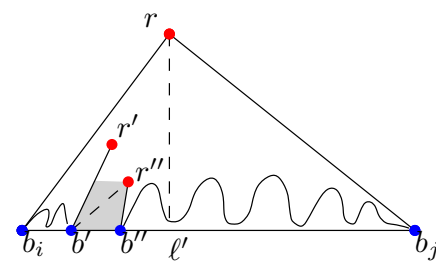

(a)

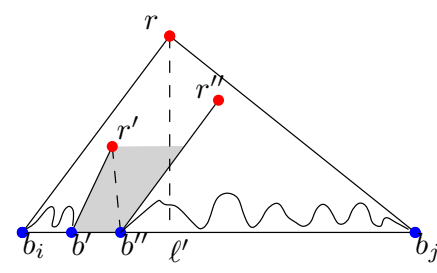

(b)

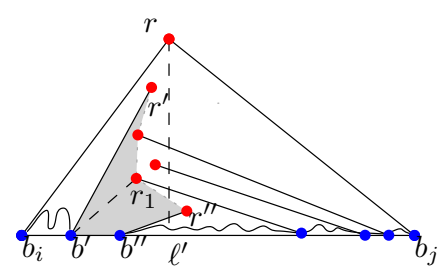

(c)

Figure 25: Illustration of proof of Lemma 3.4.1 case 2. (a) Case a: $r^{\prime}$ and $r^{\prime \prime}$ lie on the same side of $\ell^{\prime}$. (b) Case b: $r^{\prime \prime}$ lies on the other side of $\ell^{\prime}$ than $b^{\prime \prime}$ and $r^{\prime \prime}$ is higher than $r^{\prime}$. (c) Case c: $r^{\prime \prime}$ lies on the other side of $\ell^{\prime}$ than $b^{\prime \prime}$ and $r^{\prime}$ is higher than $r^{\prime \prime}$.

In case 2(b) (Figure 25 (b)), let $r^{\prime}$ be the red endpoint of the rightmost edge with endpoint $b^{\prime}$. Let $r^{\prime \prime}$ be the red endpoint of the leftmost edge with endpoint $b^{\prime \prime}$ that crosses $\ell^{\prime}$. Then, $r^{\prime} b^{\prime \prime}$ must be free, otherwise we contradict our choice of $r^{\prime} b^{\prime}$ being the rightmost edge with endpoint $b^{\prime}$. Hence, we can add the edge $r^{\prime} b^{\prime \prime}$ and remove $r b_{i}$, forming a MinBPST with less total edge length than $T^{*}$, since $\left|r^{\prime} b^{\prime}\right|<\left|r b_{i}\right|$, as $r^{\prime} b^{\prime}$ lies within the right angle triangle bounded by $r b_{i}, \ell$ and $\ell^{\prime}$, contradicting $T^{*}$ 's optimality.

In case 2(c) (Figure 25 (c)), let $r^{\prime}$ and $r^{\prime \prime}$ be the same points as defined in case 2(b). Try to add the edge $r^{\prime} b^{\prime \prime}$. If $r^{\prime} b^{\prime \prime}$ is not free, there must be at least one red point that lies to the left of $\ell^{\prime}$ and is to the right of and below $r^{\prime} b^{\prime}$. Consider all such points. Take the convex hull of these red points and $r^{\prime \prime}$. One of the red points, call it $r_{k}$, on the convex hull, must be visible by $b^{\prime}$. Add the edge $r_{k} b^{\prime}$. In either the case of adding $r^{\prime} b^{\prime \prime}$ or $r_{k} b^{\prime}$, we can remove $r b_{i}$ and form a more optimal MinBPST than $T^{*}$ contradicting the minimality of $T^{*}$. 


\subsection{Conclusion}

In this section we have described and proven that specific properties of $T^{*}$ must hold when $T^{*}$ is constructed from a semi-collinear point set.

We saw that split points must exist in $T^{*}$. Formally, there must exist two consecutive blue split points $b_{k}$ and $b_{k+1}$ such that $b_{i+1}, \ldots, b_{k}$ are $\bar{r}$-connected to $b_{i}$, but not $\bar{r}$ connected to $b_{j}$ and $b_{k+1}, \ldots, b_{j-1}$ are $\bar{r}$-connected to $b_{j}$, but not $\bar{r}$-connected to $b_{i}$.

We proved if $\max \left\{\angle r b_{i} b_{j}, \angle r b_{j} b_{i}\right\} \geq 90^{\circ}$, then there is no red point in the triangle $\triangle r b_{i} b_{j}$. Furthermore, in such a triangle if any blue points lie between $b_{i}$ and $b_{j}$, they must be adjacent to $r$ in $T^{*}$.

Finally, we showed that if $b_{1}$ and $b_{n}$ are not split points then there are no $\ell^{\prime}$-crossing edges. That is to say that no edge crosses the line perpendicular to $\ell$ that goes through $r$.

It is possible to use these properties in the design and implementation of the dynamic programming algorithm. However, the running time does not improve. 


\section{Chapter 4}

\section{Conclusion and Future Work}

In this chapter we summarize the results described in this thesis and outline areas for potential future research.

\subsection{Contributions}

In this thesis we described an $O\left(|B|^{3}|R|^{2}\right)$ algorithm that finds a MinBPST for a set of bichromatic semi-collinear points and its implementation. Additionally, we showed how this algorithm can be adapted to find a MaxBPST, a minimum bottleneck bichromatic plane spanning tree, and a maximum bottleneck bichromatic plane spanning tree on a set of bichromatic semi-collinear points. Finally, we described properties of a MinBPST, $T^{*}$, on a set of semi-collinear points. These properties may prove to be useful by future researchers.

\subsection{Future Work}

The following related problems require future research:

- Given a bichromatic plane spanning tree is there an efficient way to determine if 
the tree is in fact a minimum bichromatic plane spanning tree?

- Borgelt et al. [10] presented an approximation algorithm for finding the MinBPST with a $O(\sqrt{n})$ approximation ratio for the general case. Is it possible to find a constant factor approximation algorithm that runs in polynomial time?

- Chapter 3 describes properties of a MinBPST on semi-collinear point sets. Is it possible to use these properties to create an algorithm for finding a MinBPST on a set of semi-collinear points that has a running time better than $O\left(|B|^{3}|R|^{2}\right)$ ?

- Given a set of blue points on a line, $\ell$, and a set of red points on one side of the line, what is the ratio between the minimum bichromatic spanning tree and the minimum bichromatic plane spanning tree? We have found that the ratio is at least $\frac{3}{2}$. Take two blue points: $b_{1}$ located at $(0,0)$ and $b_{2}$ located at at $(0,1)$, and two red points: $r_{1}$ located at $(-\cos \alpha, \sin \alpha)$ and $r_{2}$ located at $\left(-\epsilon, \frac{1}{2} \epsilon \tan \alpha\right)$, where $\alpha$ is the angle between $\ell$ and $r_{1} b_{1}$. We can minimize the angle $\alpha$ and the value of $\epsilon$. See Figure 26 for more detail; (a) shows the MinBPST and (b) shows the minimum bichromatic spanning tree. Note that this illustration is not to scale and $\alpha$ and $\epsilon$ are not minimized so that they are large enough for the reader to see. Altogether we see that the ratio must be at least $\frac{3}{2}$. We ran experiments on randomized point sets (with blue points on a line and red points to one side of the line) comparing the length of the MinBPST found by our algorithm to the length of the minimum bichromatic spanning tree found by running Kruskal's algorithm on the complete bipartite graph of the point set where the points are partitioned by colour. We never obtained a value larger than $\frac{3}{2}$. In fact in our experimentation on random point sets we never came close to this value; most of our resulting ratios were close to 1 . Thus, we conjecture that the ratio is $\frac{3}{2}$, but it has yet to be proven. 


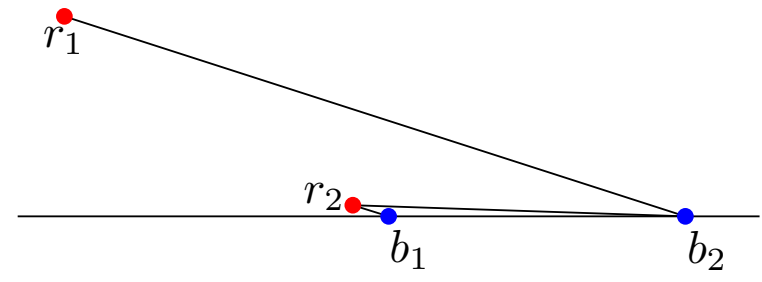

(a)

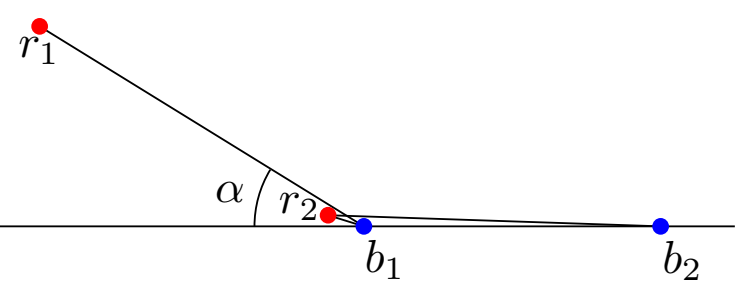

(b)

Figure 26: An illustration to show a potential approximation ratio between the MinBPST and the minimum bichromatic spanning tree. (a) MinBPST (b) Minimum Bichromatic Spanning Tree 


\section{Bibliography}

[1] A. Kaneko and M. Kano. "Discrete geometry on red and blue points in the planea survey-." In "Discrete and computational geometry," pages 551-570. Springer (2003).

[2] R. C. Prim. "Shortest connection networks and some generalizations." Bell system technical journal 36(6), 1389-1401 (1957).

[3] J. Nešetřil, E. Milková, and H. Nešetřilová. “Otakar boruvka on minimum spanning tree problem translation of both the 1926 papers, comments, history." Discrete mathematics 233(1), 3-36 (2001).

[4] J. B. Kruskal. "On the shortest spanning subtree of a graph and the traveling salesman problem.” Proceedings of the American Mathematical society 7(1), 4850 (1956).

[5] O. Boruvka. “O jistém problému minimálním.” (1926).

[6] F. P. Preparata and M. Shamos. Computational geometry: an introduction. Springer Science \& Business Media (2012).

[7] P. Bose and G. Toussaint. “Growing a tree from its branches.” Journal of Algorithms 19(1), 86-103 (1995).

[8] A. Biniaz, P. Bose, D. Eppstein, A. Maheshwari, P. Morin, and M. Smid. "Spanning trees in multipartite geometric graphs.” arXiv preprint arXiv:1611.01661 (2016).

[9] M. Grantson, H. Meijer, and D. Rappaport. "Bi-chromatic minimum spanning trees.” In “EuroCG,” pages 199-202 (2005).

[10] M. G. Borgelt, M. Van Kreveld, M. Löffler, J. Luo, D. Merrick, R. I. Silveira, and M. Vahedi. "Planar bichromatic minimum spanning trees." Journal of Discrete Algorithms 7(4), 469-478 (2009). 
[11] D. Lichtenstein. "Planar formulae and their uses." SIAM journal on computing 11 (2), 329-343 (1982). 